\title{
The Functionality of a Novel Robotic Surgical Assistant for Total Knee Arthroplasty: A Case Series
}

\author{
Alexandra Alessi $\mathbb{D}^{1,2}$ Evan Fitzcharles $\mathbb{D}^{1,2}$ Ian C. Weber $\mathbb{D}^{3},{ }^{3}$ and Nathan L. Cafferky $\mathbb{D}^{2,3}$ \\ ${ }^{1}$ Vail Summit Orthopaedic Foundation, 181 West Meadow Dr., Suite 2700, Vail, CO, USA 81657 \\ ${ }^{2}$ Vail Summit Orthopaedics and Neurosurgery, 181 West Meadow Dr., Suite 2700, Vail, CO, USA 81657 \\ ${ }^{3}$ Cornerstone Orthopaedics and Sports Medicine, $500 \mathrm{~W}$ 144th Avenue, Suite 230, Westminster, CO, USA 80023
}

Correspondence should be addressed to Nathan L. Cafferky; ncafferky@vsortho.com

Received 18 November 2020; Revised 15 February 2021; Accepted 23 February 2021; Published 17 March 2021

Academic Editor: Werner Kolb

Copyright () 2021 Alexandra Alessi et al. This is an open access article distributed under the Creative Commons Attribution License, which permits unrestricted use, distribution, and reproduction in any medium, provided the original work is properly cited.

\begin{abstract}
Conventional total knee arthroplasty and soft tissue balancing is based on a subjective unquantified assessment, which can lead to imperfect balancing and poor patient outcomes. Five case studies were used to present the functionality of a novel robotic system in allowing intraoperative adjustments based on objective measures for several primary total knee arthroplasty cases. The robotic system allows the surgeon to drive every step of the case, turning the subjective nature of conventional knee replacement into a more objective and scientific approach for restoration of alignment, gap balancing, joint space restoration, femoral rotation, and Q-angle restoration. The robotic system allowed precise intraoperative adjustments, as demonstrated by these cases, and is a promising step towards more personalized total knee arthroplasty made possible by utilizing real-time objective measures.
\end{abstract}

\section{Introduction}

The patient-reported dissatisfaction rate for primary total knee arthroplasty (TKA) is approximately $10 \%$ to $20 \%[1-$ 3]. However, the introduction of robotic technology has the potential to change the narrative in TKA outcomes by allowing for greater precision in implant positioning compared to manual instrumentation [4-8]. Further, recent studies have suggested robotic-assisted TKA (RATKA) patients experience faster short-term recovery than conventional patients $[9,10]$. Unfortunately, they have failed to show navigation or robotics improving long-term results [11-13]. Despite this, recent data has shown RATKA to be advantageous in correcting knee alignment intraoperatively compared with conventional instrumentation [14]; however, there is a lack of literature demonstrating the ability of novel robotic systems to achieve neutral mechanical alignment throughout varying degrees of deformity. For instance, patients with severe varus or valgus deformity present greater difficulty in balancing the coronal plane of the knee.
In the development of robotic systems used in TKA procedures, it is crucial for the systems to recognize and register a patient's key anatomical landmarks found on the femur, tibia, and ankle. This information is historically obtained using either preoperative advanced imaging CT or MRI or a combined system of radiographs (image-based) or by simply using intraoperative bone mapping (image-free) [15] New X-ray-based preoperative planning is available that allows for 3-dimentional image processing from 2dimentional plain film radiographs (X-Atlas ${ }^{\mathrm{TM}}$, Zimmer Biomet, USA). These systems negate the need for expensive preoperative advanced imaging studies and are a more cost conscious approach to preoperative templating and robotic TKA. The preoperative templating with weight-bearing imaging may provide greater accuracy for alignment than intraoperative navigation alone, and the robotic system used in these cases allows for both image-based and image-free work flows. Robotic systems aid the surgeon in creating a patient-specific model and registering the model and patient's anatomical landmarks intraoperatively, ultimately 


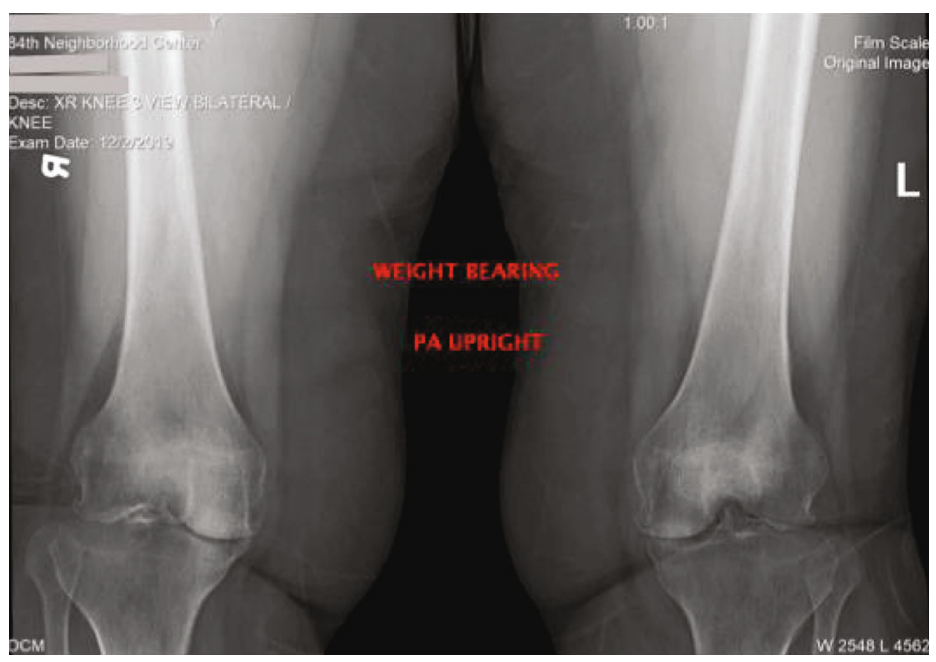

(a)

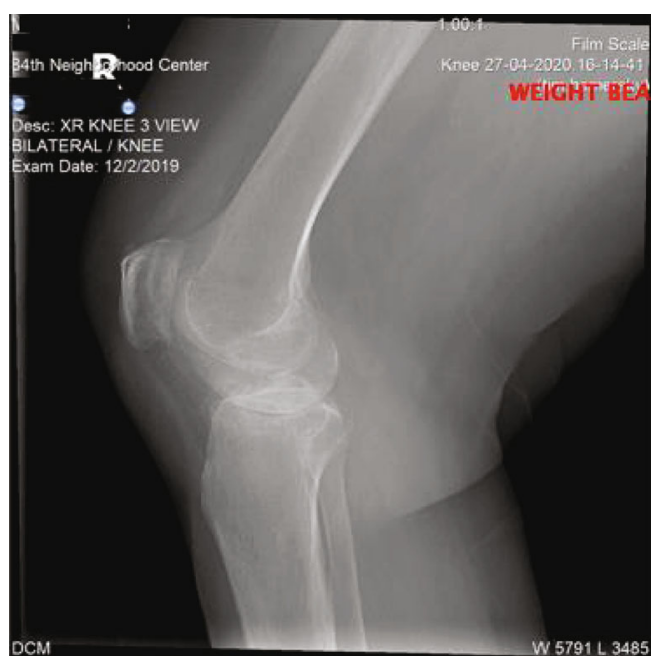

(b)

FIgure 1: Preoperative weight bearing AP (a) and lateral (b) X-rays for Case 1.

TABLE 1: The range of motion at maximum flexion and extension in the initial and final evaluations using the robotic knee application.

\begin{tabular}{ccccc}
\hline \multirow{2}{*}{ Case } & \multicolumn{2}{c}{ Initial } & \multicolumn{2}{c}{ Final } \\
& Extension $\left(0^{\circ}\right)$ & Flexion $\left(90^{\circ}\right)$ & Extension $\left(0^{\circ}\right)$ & Flexion $\left(90^{\circ}\right)$ \\
\hline 1 & $5^{\circ}$ & $102^{\circ}$ & $2^{\circ}$ & $127^{\circ}$ \\
2 & $1^{\circ}$ & $129^{\circ}$ & $5^{\circ}$ & $127^{\circ}$ \\
3 & $1.5^{\circ}$ & $122^{\circ}$ & $5.5^{\circ}$ & $131^{\circ}$ \\
4 & $9^{\circ}$ & $110^{\circ}$ & $0.5^{\circ}$ & $137^{\circ}$ \\
5 & $23^{\circ}$ & $126^{\circ}$ & $0.5^{\circ}$ & $141^{\circ}$ \\
\hline
\end{tabular}

helping carry out bone resection, implant positioning, and limb alignment [16].

A novel semiautonomous robotic-arm surgical assistant has recently been introduced which incorporates the robotic placement of cutting jigs and includes a ligament balancing evaluation tool. This is unlike other systems that involve either a passive mode, where the surgeon holds the bone mill, or an active mode, where the surgeon is not physically engaged in the milling process at all [17]. It is also different from the haptic arm, which limits the surgeon to predetermined boundaries [4]. To our knowledge, there have been no clinical reports demonstrating the use or outcomes of this system. As such, the purpose of this small case series is to present several primary TKA cases $(n=5)$ and the functionality of this system to address intraoperative adjustments in simple and complex cases.

\section{Case Presentations}

The robotic surgical assistant used in each case was the ROSA $^{\circledR}$ Total Knee System (Zimmer Biomet Warsaw, Indiana, USA). The system incorporates two components: (1) a robotic arm and base unit are positioned on the same side as the surgeon, and (2) a camera unit is positioned on the contralateral side of the patient.
With this system, two rigid body trackers allow for triangulation of the knee joint between the robotic base unit and the camera unit. Thirty-six anatomical landmark data points are obtained in order to map out the intra-articular anatomy. This enables the robot to identify the knee in space and allows the surgeon to make real-time intraoperative, patient-specific adjustments according to the patient's unique anatomy. The robotic arm assists in positioning the cutting jigs in the planned cutting plane and allows for accuracy down to $0.5 \mathrm{~mm}[7,18]$.

For each case, the patient reported to the operating theatre and was placed in the supine position with the robotic arm placed on the ipsilateral side of the patient along with the surgeon. The optical tower was placed opposite of that. Following standard procedures, the knee was cleaned and prepped followed by exposure to the joint using a medial parapatellar approach. Consent was obtained from each patient in order to be included in this case report.

2.1. Case 1: Image-Free TKA. The functionality of this robotic system provides for both image-based and image-free surgeries. This case helps illustrate the use of the image-free application to restore a neutral mechanical alignment. A 67-yearold female presented with severe osteoarthritis; KellgrenLawrence [19] grade 4 changes on radiographic review (Figure 1). During the initial knee evaluation performed using the robotic application prior to performing any boney cuts (hereinafter, initial knee evaluation), the knee behaved varus at extension and the knee was not able to reach a full range of motion (Table 1). The goal was to restore medial to lateral stability by balancing the tibiofemoral gap via bone resections and soft tissue balancing and to correct the varus deformity throughout the range of motion by balancing the variation in bone gaps. The initial evaluation showed lateral laxity in extension and a larger medial space in flexion with an overall tighter tibiofemoral gap at $90^{\circ}$ (Table 2). Reducing lateral joint spacing in extension is in order to release tension medially where the soft tissue is tight and to create 
TABLE 2: The tibiofemoral lateral and medial gap spacing $(\mathrm{mm})$ in both extension and flexion in the initial evaluation of surgery as well as in the final evaluation following bone resections when varus and valgus stress was applied, respectively.

\begin{tabular}{lcccccccc}
\hline & & \multicolumn{2}{c}{ Initial } & \multicolumn{2}{c}{ Flexion $\left(90^{\circ}\right)$} & \multicolumn{2}{c}{ Extension $\left(0^{\circ}\right)$} & Final \\
\hline Case & Lateral & Medial & Lateral & Medial & Lateral & Medial & Lateral \\
1 & 5.5 & 3.0 & 2.5 & 3.5 & 1.0 & 1.0 & 1.5 \\
2 & 4.5 & 1.5 & 5.0 & 4.0 & 1.0 & 1.0 & 1.0 & 0.5 \\
3 & 6.0 & 2.5 & $\mathrm{~N} / \mathrm{A}^{1}$ & 7.5 & 2.0 & 2.0 & 1.0 \\
4 & 6.0 & 4.5 & 11.5 & 3.5 & 1.0 & 1.0 & 1.0 & 1.0 \\
5 & 3.5 & 5.0 & 4.0 & 8.5 & 1.5 & 1.5 & 1.5 \\
\hline
\end{tabular}

N/A: not assessed.

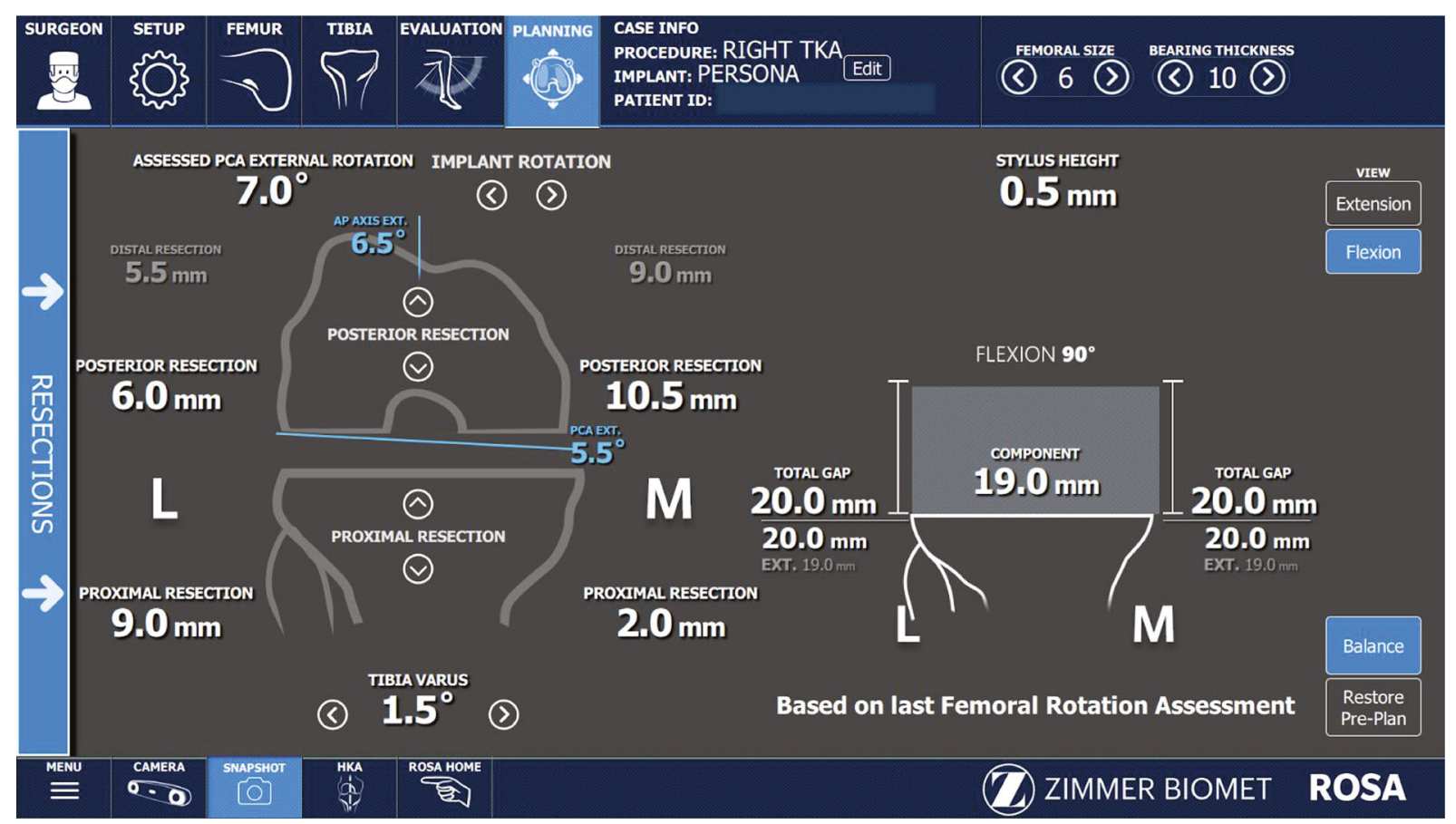

FIgURE 2: An intraoperative surgeon generated plan for femoral resections of Case 1.

appropriate tension in lax lateral ligaments to correct the knee out of its varus alignment. As such, the initial surgical plan was to minimize the resection on the proximal tibia medially $(2.0 \mathrm{~mm})$ and resect the necessary amount of bone from the posterior medial femur $(10.5 \mathrm{~mm})$ to achieve the alignment goals (Figure 2). A medial tibial reduction osteotomy [20], as well as the removal of the lateral osteophytes, was used to address the varus knee behavior by releasing tension on tight medial ligaments. The use of the appropriate polyethylene bearing thickness was also used to tighten the laxity in the medial and lateral ligaments (Table 2).

Ultimately, the surgeon was able to restore the full range of motion (Table 1), and the patient was left slightly varus with a balanced ligamentous gap between the femoral and tibial implants of approximately one millimeter on each side (Table 2).

This image-free case, relying on intraoperative anatomic land marking and soft tissue assessment at the initial knee evaluation stage, resulted in a neutral mechanical alignment during final evaluation. Though alignment cannot be accurately measured on short film X-rays, these postoperative $\mathrm{X}$-rays are useful in demonstrating a uniform tibiofemoral gap and well aligned femoral and tibial components (Figure 3). The gap balancing measures by the robotic system (Table 2) are detailed in the surgeon report, eliminating the need to rely on postoperative X-rays to ensure the knee is balanced and well aligned.

2.2. Case 2: Gap Balance and Femoral Rotation. A 57-yearold male presented with worsening right knee pain, and preoperative X-rays showed tricompartmental arthritis consistent with Kellgren-Lawrence [19] grade 3 changes (Figure 4). The preoperative physical exam showed full knee range-of-motion (ROM).

The initial intraoperative knee evaluation was performed (Figure 5), evaluating the knee range of motion and alignment (Figure 5(a)) and coronal stability, represented by the blue shaded area, to varus and valgus stress at $0,30,45$, and 


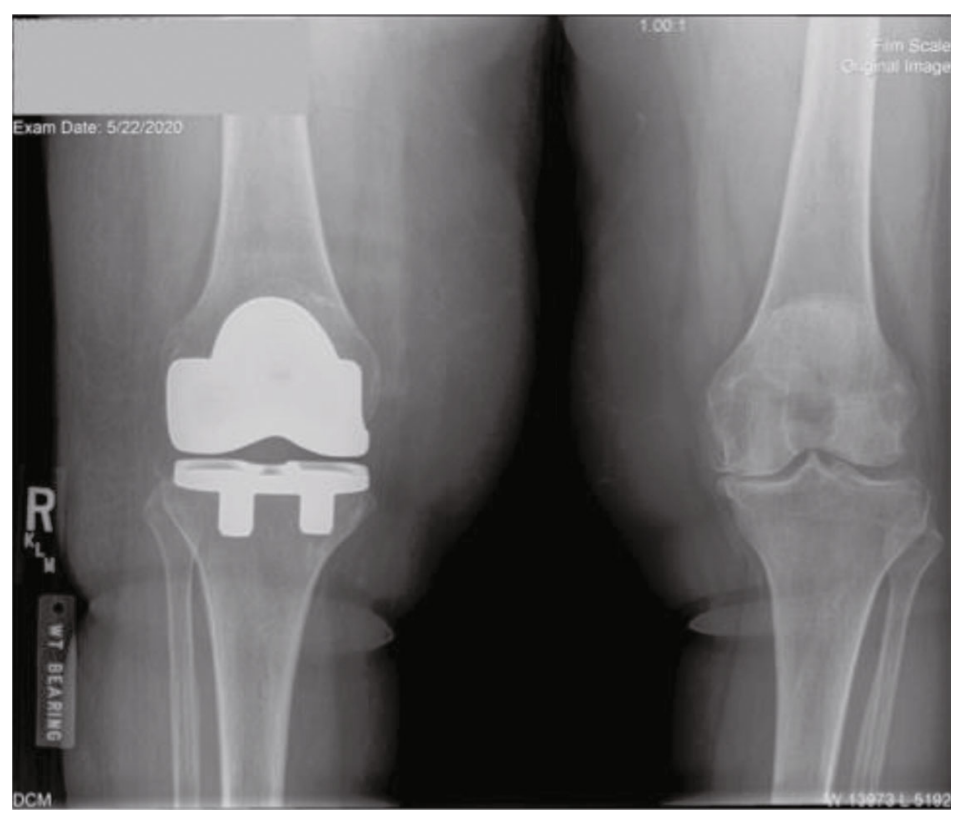

(a)

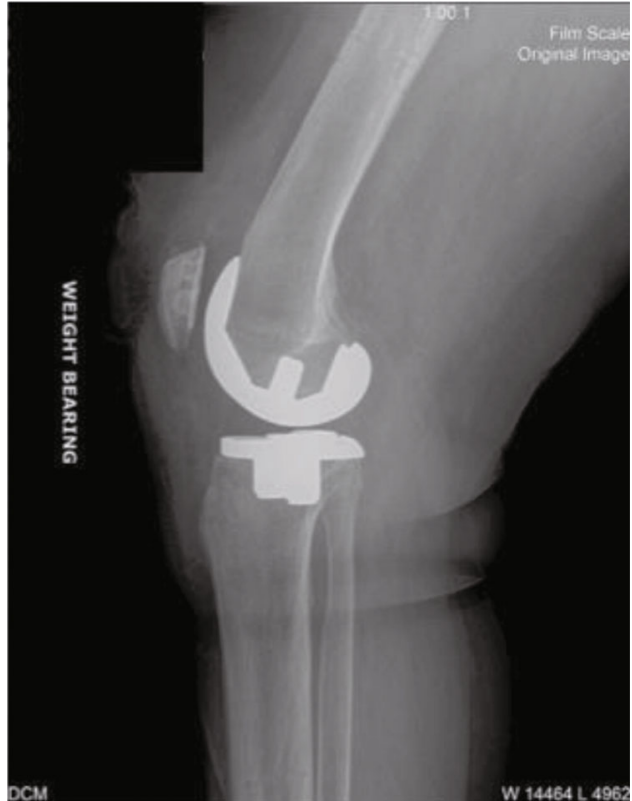

(b)

Figure 3: Postoperative AP (a) and lateral (b) X-rays of the right knee for Case 1.

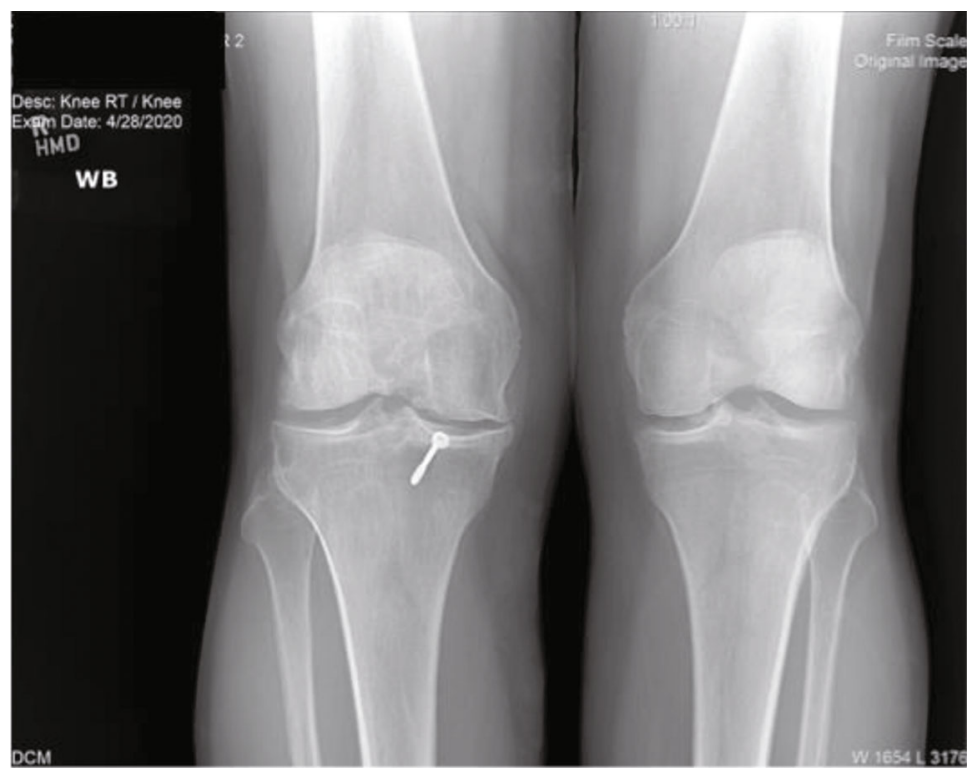

(a)

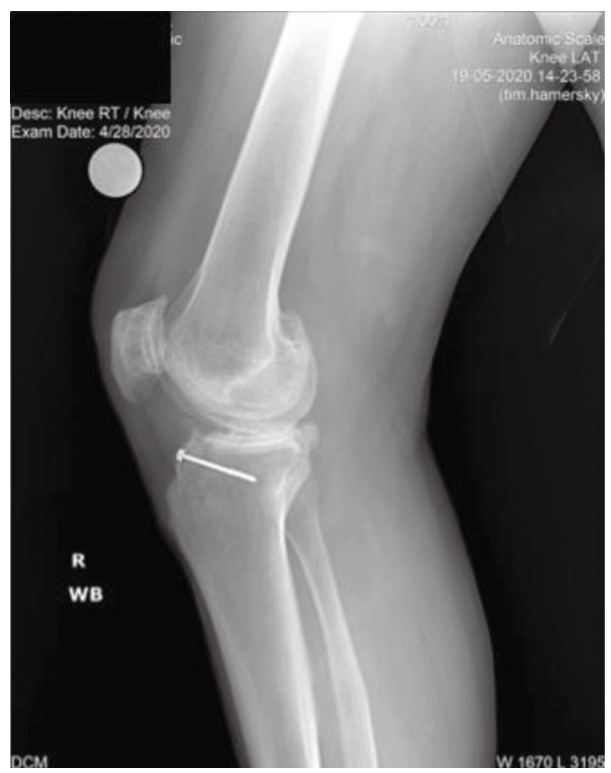

(b)

FIgURE 4: Preoperative weight bearing radiographs of Case 2 showing the tricompartmental arthritic changes of the right knee on the (a) anteroposterior view and (b) the arthritic changes of the knee on the lateral view.

90 degrees for knee ROM (Figure 5(b)). The measurements from the knee evaluation panel of the robotic system for this case showed the right knee presented with slight valgus measures in extension and a range of $2.2^{\circ}$ valgus to $0.9^{\circ}$ varus, but corrected into varus throughout the knee range of motion. It should be noted that this technology allows a surgeon to evaluate varus and valgus stability of the operative knee at 0,30 , $45,60,90$, and 120 degrees of knee ROM, but it is the preference of this surgeon to only evaluate varus and valgus stress at $0,30,45$, and 90 degrees. A numerical assessment of the extension gap at 0 degrees, and flexion gap at 90 degrees, prior to any boney resections is provided (Figure 5(c)). As illustrated, the medial knee joint is tighter than the lateral knee joint. These numbers are obtained by stressing the knee to varus and valgus forces at the initial knee state evaluation, as seen in Figure 5(b). The initial knee state evaluation is critical to obtain, as it allows the surgeon and robotic assistant to better understand the soft envelope around the arthritic knee 


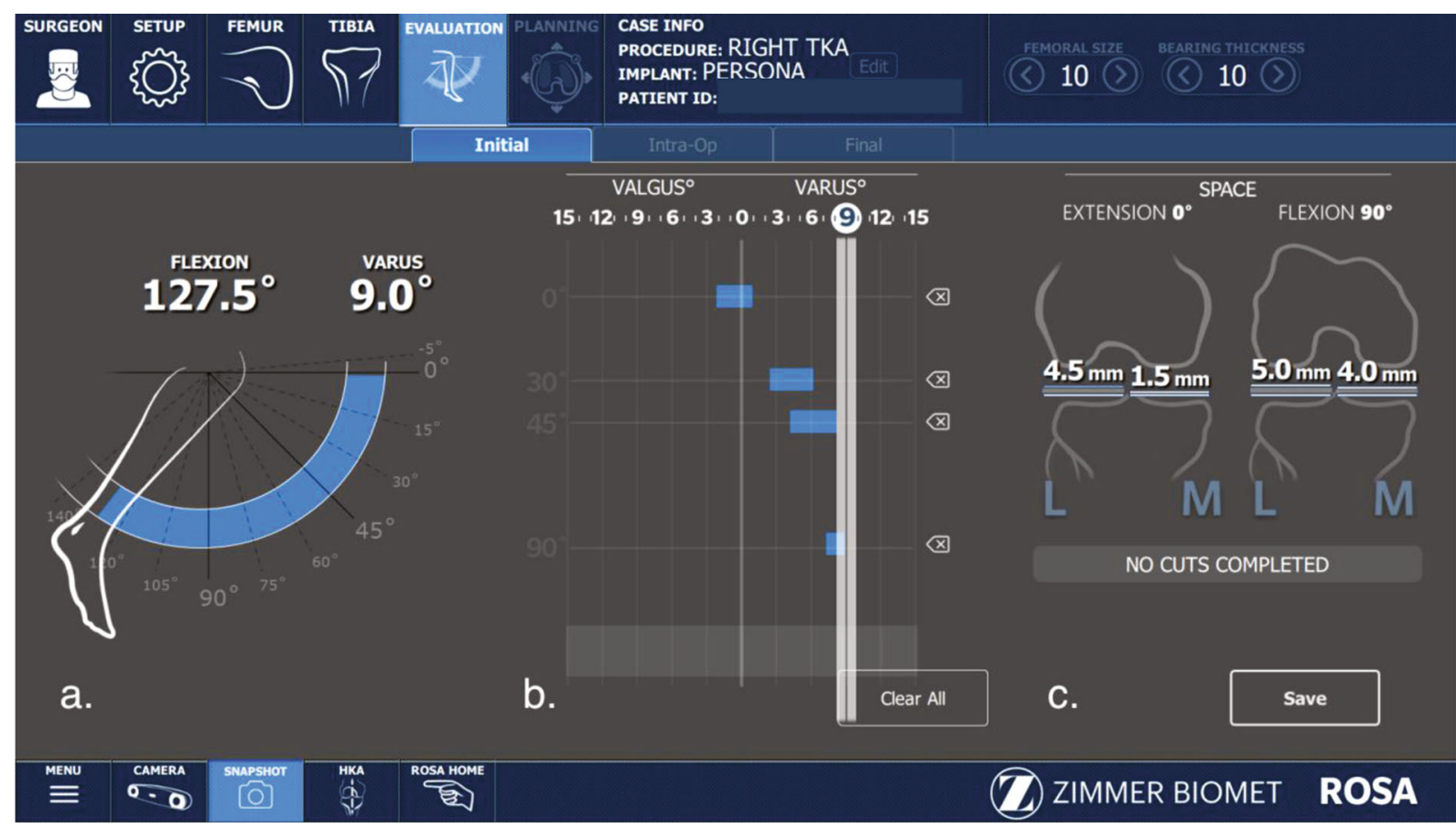

FIGURE 5: The robotic system's knee evaluation tool in the initial stage of Case 2, which includes a preoperative evaluation of range of motion, varus/valgus alignment, and tibiofemoral joint gap spacing in flexion and extension.

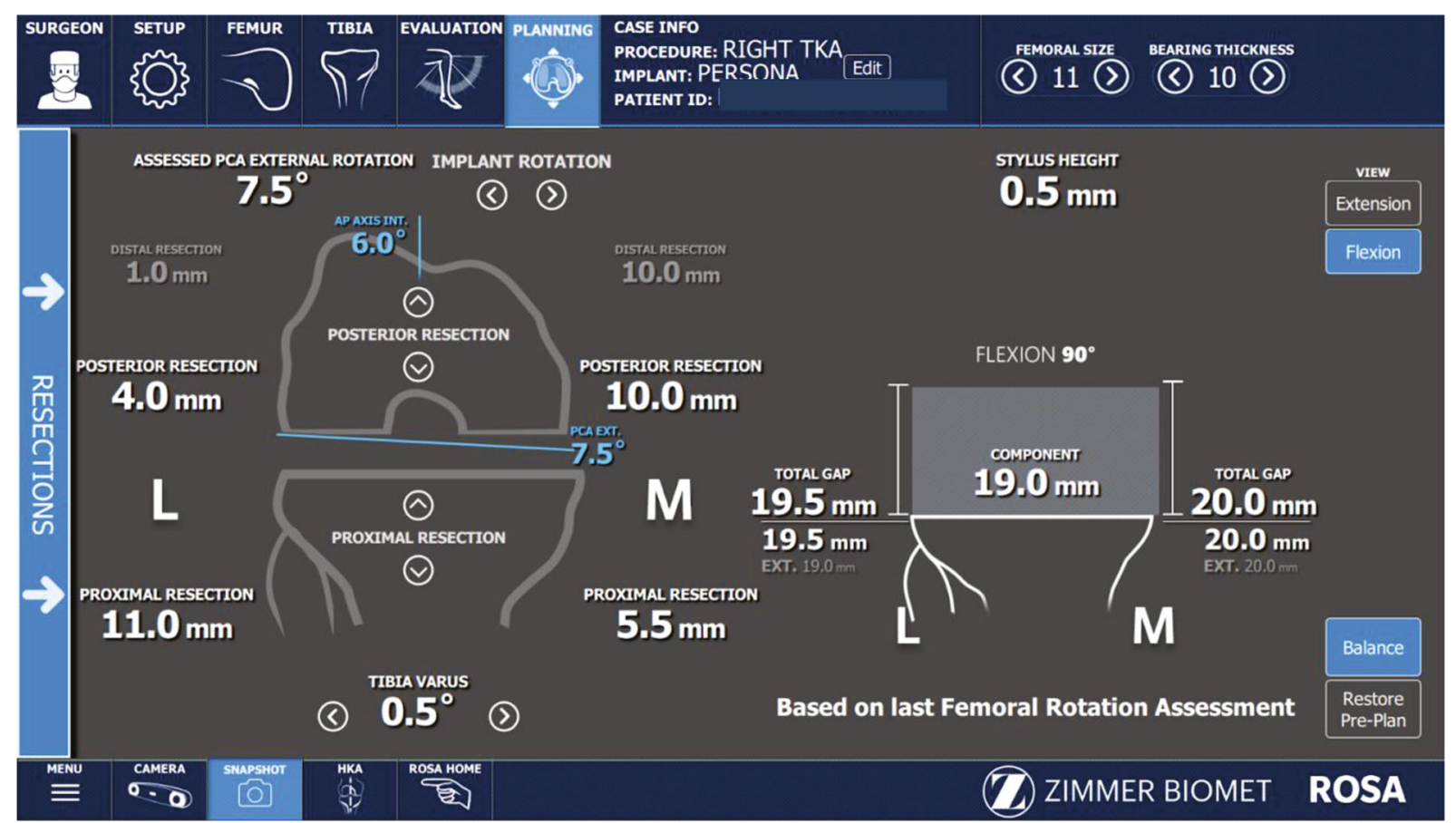

Figure 6: An intraoperative generated plan of Case 2 demonstrating planned femoral and tibial resections.

joint. This gives the surgeon a more objective approach to each additional downstream step in the total knee replacement procedure and helps guide additional boney resections or soft tissue releases, as the information becomes available.

The goal was to balance the mechanical and anatomical axis of the knee and improve the patient's knee kinematics.
Contrary to conventional instrumentation, the robot guided the accuracy of the bone resections. An image-free view of the knee is illustrated by a schematic knee drawing (Figure 6). The digital view of the knee can be easily changed between an extension view and a flexion view of the knee joint. When the knee is in 90 degrees of flexion, the surgeon 


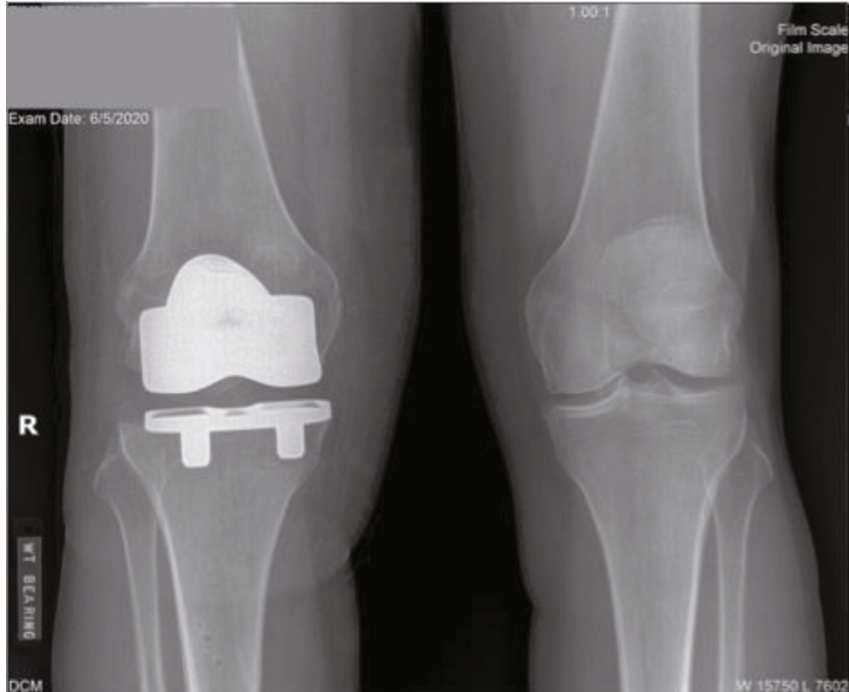

(a)

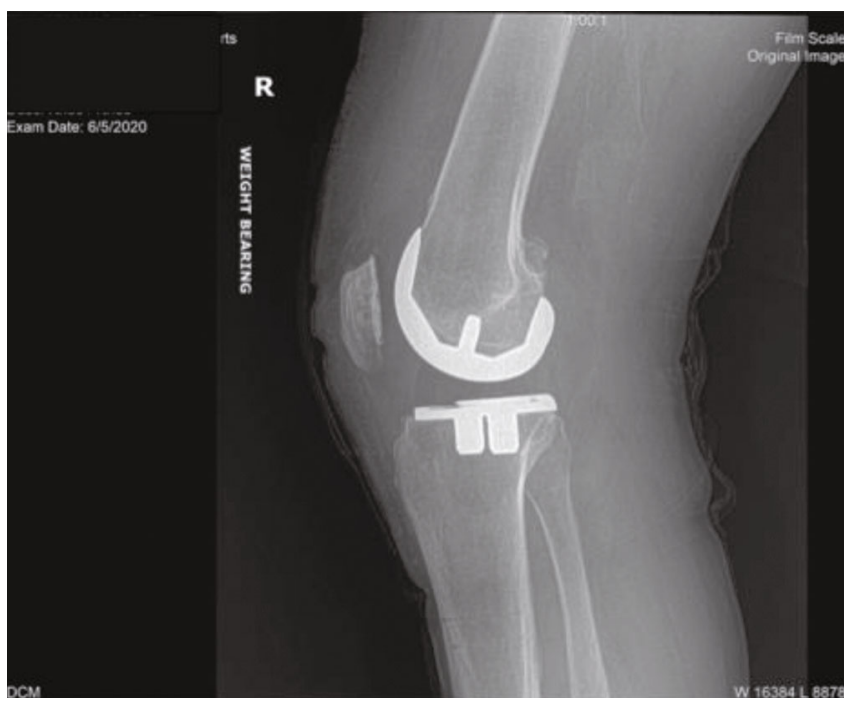

(b)

FIgURe 7: Postoperative weight bearing AP (a) and lateral (b) X-rays showing the final alignment and joint spacing of the right knee for Case 2.

has an axial view of the distal femur, allowing the surgeon to appreciate the femoral rotation (Figure 6). In this case, the femur is externally rotated to 7.5 degrees of the posterior condylar axis, which is more than the conventional instrumentation of 3 and 5 degrees of external rotation (Figure 6). The view of the tibia is also appreciated in the schematic of the knee (Figure 6). The plan is to leave the tibia in 0.5 degrees of varus alignment, with anticipated medial and lateral tibial resections of $5.5 \mathrm{~mm}$ and $11.0 \mathrm{~mm}$, accordingly. All measurements were obtained by the robot using an optical tracking system. These measurements were used to guide the plan for resection, allowing for the appropriate space and positioning of the implant. Tibial resection was performed to achieve a balanced extension gap in coronal alignment (Figure 6). It is also important to mention that after every boney resection the surgeon must validate the accuracy of each cut before allowing to move on. With the sclerotic bone,

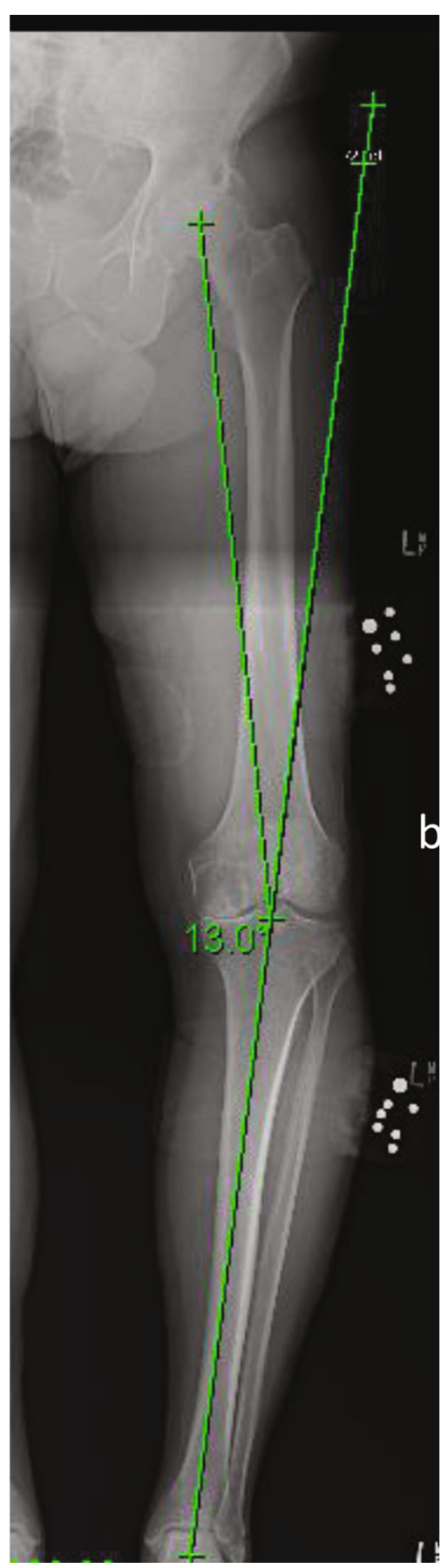

Figure 8: Preoperative long-leg X-ray showing the weight-bearing functional alignment with 13.0 degrees of varus and osteoarthritis of the left knee for Case 3.

the saw blade could skive and give you an erroneous resection. If not identified, this could alter the lower extremity alignment and soft tissue balance at the end of the case. The robotic assistant does allow for a 1.5 degree and $1.5 \mathrm{~mm}$ of tolerance for every step, and it is up to the surgeon to decide whether to accept the validation or not. If an unacceptable resection were to be performed, the robotic assistant easily allows the surgeon to recut. It is imperative that the surgeon remember to fall back on their training, as a safety 


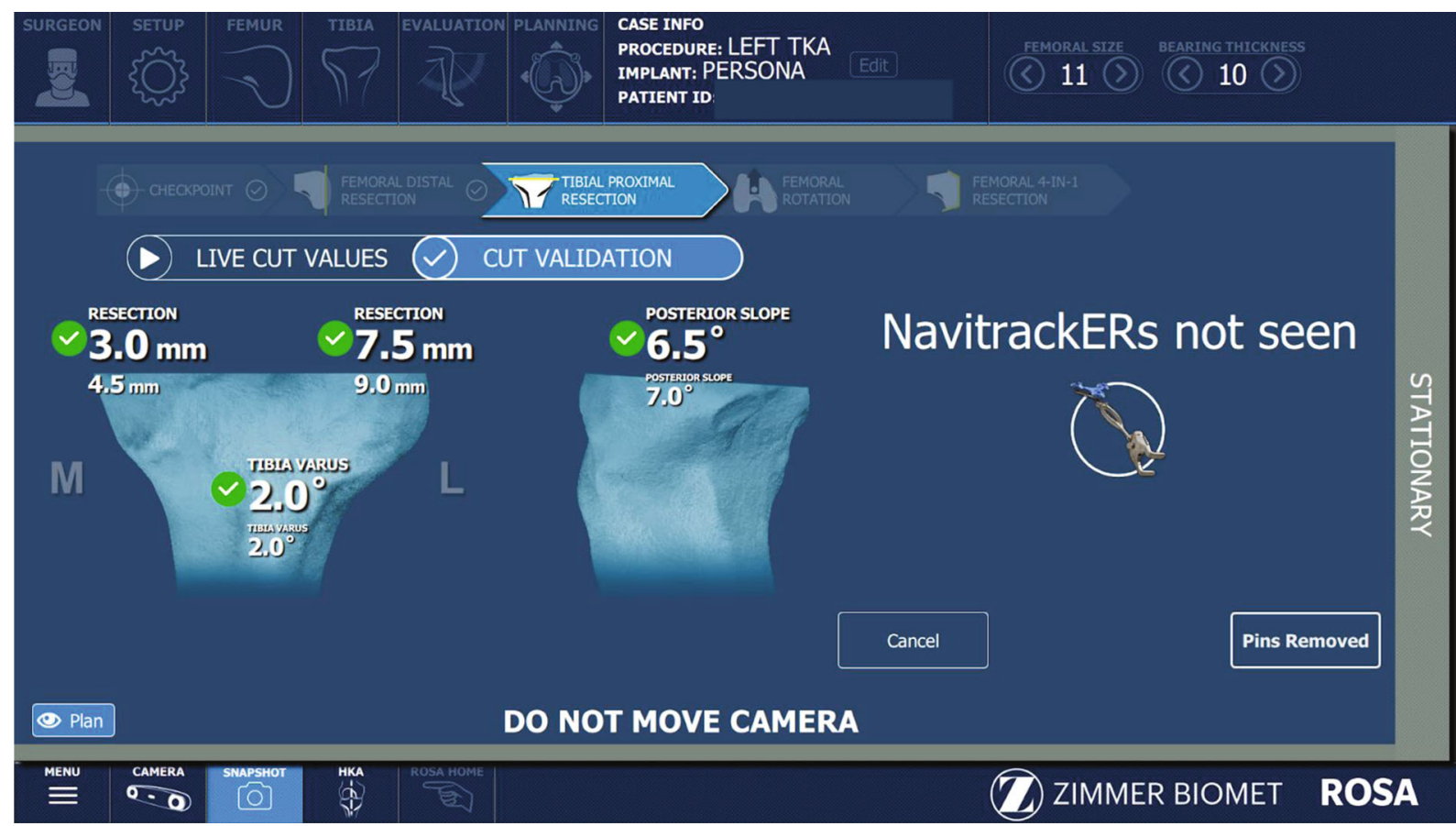

FIgURE 9: Final proximal tibial resections validated against the planned resections for Case 3.

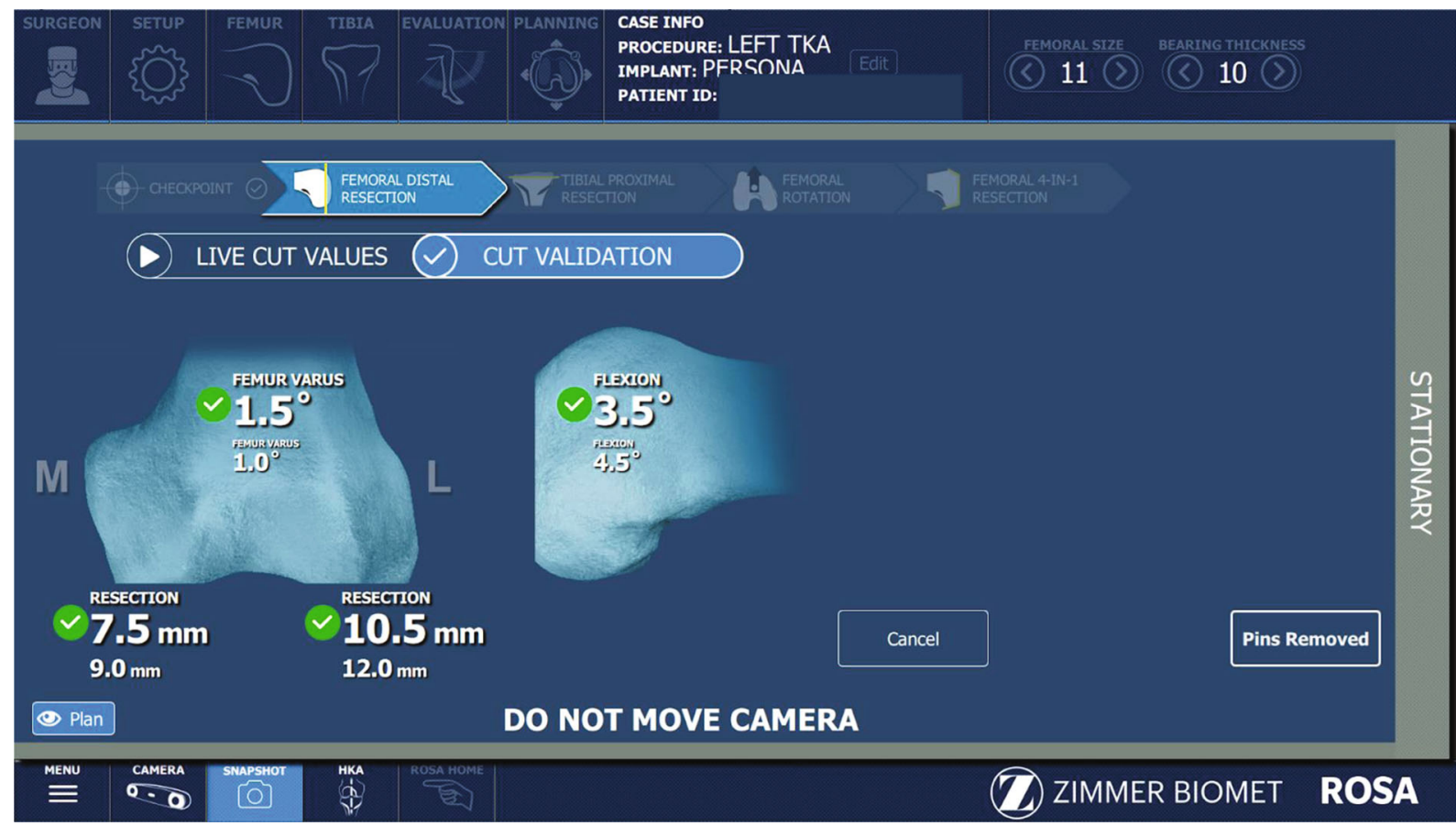

Figure 10: Final distal femoral resections validated against planned resections for Case 3.

measure. With this case, the result allowed for restoration of alignment, gap balancing, femoral rotation, and joint restoration with as little soft tissue release as possible.

The primary goal for this moderately degenerative knee was performing a TKA that restored the alignment of the knee from a varus alignment of $9.0^{\circ}$ in flexion to a more neutral state throughout the entire range of motion (Figure 5).
Correcting alignment and restoring the patient's knee kinematics are necessary to ensure success for the lifetime of the implant [21-24]. A misaligned implant will result in stress being placed on implant bearing surfaces as well as boneimplant interfaces. A properly aligned implant will also aid in the balancing of the forces transmitted through the soft tissue. Because of the $7.5^{\circ}$ external rotation of the PCA axis, the 
application provided a plan for resection that allowed the surgeon to align the rotation by resecting more from the medial posterior side of the femur with $10.0 \mathrm{~mm}$ while only cutting $4.0 \mathrm{~mm}$ from the lateral side (Figure 6).

Finally, upon placement of the final implant and prior to closure, the surgeon reevaluated the knee in a similar manner to the initial phase in order to obtain the final knee evaluation data. A neutral mechanical axis was restored at extension while maintaining full range of motion (Table 1, Figure 5(a)), increasing the coronal stability of the knee (Figure 5(b)), and balancing the gap between implants (Figure 5(c)). Postoperative ROSA evaluation and radiographs show the restoration of the knee to a neutral mechanical alignment throughout the knee ROM, mirroring the patient's natural alignment of their nonoperative side (Figure 7), and a final intraoperative evaluation and postoperative physical exam findings demonstrating full ROM (Table 1).

2.3. Case 3: Alignment Correction. A 65-year-old male presented with end-stage osteoarthritis in the left knee. Preoperative weight-bearing X-rays show a severe varus deformity along with Kellgren-Lawrence [19] grade 4 changes (Figure 8). An initial evaluation of the knee showed a varus deformity in both extension and flexion. While the patient had a fair range of motion (Table 1), there was significant lateral spacing in flexion with no medial gap (Table 2), which was contributing to the severe varus alignment.

To address the severe varus deformity, proximal tibial resections were made, as well as distal femoral resections, which were validated by the system (Figures 9 and 10).

The robotic system allowed for more precise bone resections which resulted in minimal medial soft tissue releases and balanced flexion and extension gaps. Even though medial ligament tightness would normally contribute to varus deformities, these precise resections preserved the soft-tissue envelope while also ensuring balanced gaps. The tibiofemoral gap in extension was balanced medially and laterally in both extension and flexion (Table 2). The final knee evaluation showed a reduction in the severity of varus alignment in extension and flexion, with a planned overall final hip-knee-ankle angle (HKA) of $0.3^{\circ}$ varus (Figure 11). It should be noted that this patient appears to have lost 4 degrees of extension in the final knee evaluation (Table 1). However, the system evaluates the flexion with the inclusion of the femoral bow and so it is recommended that the surgeon perform a clinical evaluation to confirm the robotic measures. In this case, the patient demonstrated full extension clinically and so no further adjustments were needed.

2.4. Case 4: Surgical Correction of Knee Range of Motion. A 59-year-old male was scheduled to undergo a right TKA after presenting with severe tricompartmental osteoarthritic changes, more so in the lateral compartment with joint space narrowing, sclerosis, and osteophytes present (Figure 12). The initial knee evaluation performed intraoperatively using the robotic system showed limited knee ROM (Table 1), a strong varus deformity in flexion, significant lateral laxity, and a valgus deformity in extension.

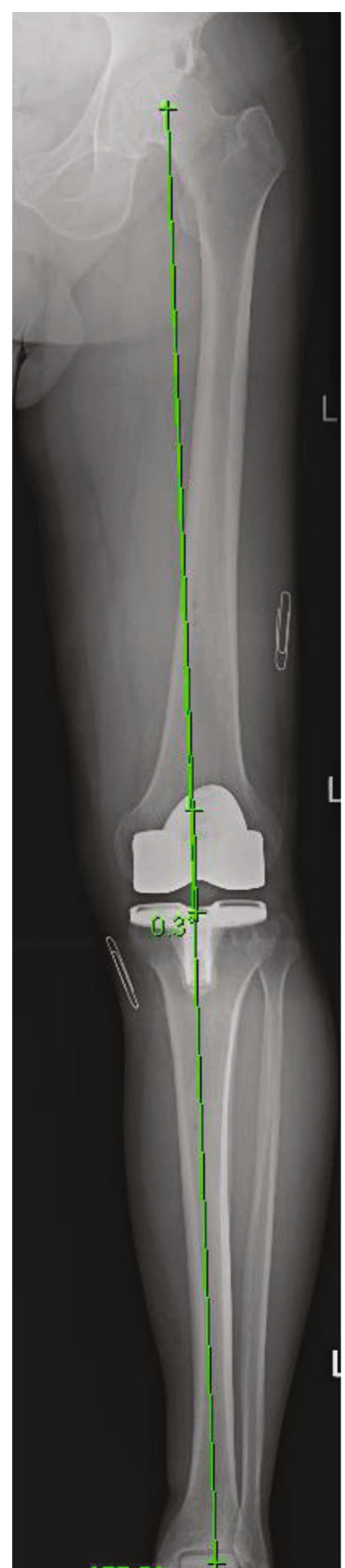

FIGURE 11: Postoperative long-leg standing radiograph of the left leg for Case 3 showing the corrected neutral mechanical alignment of $0.3^{\circ}$ varus following TKA. 


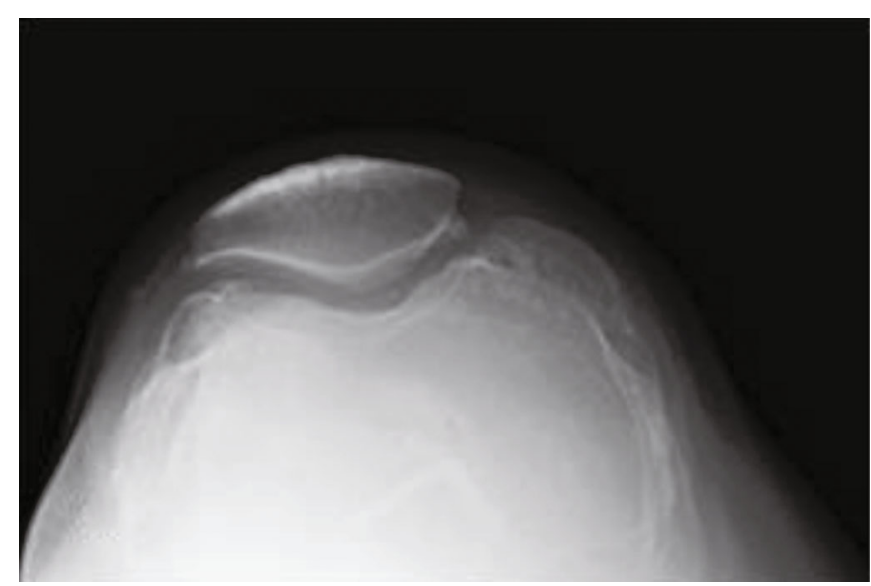

(a)

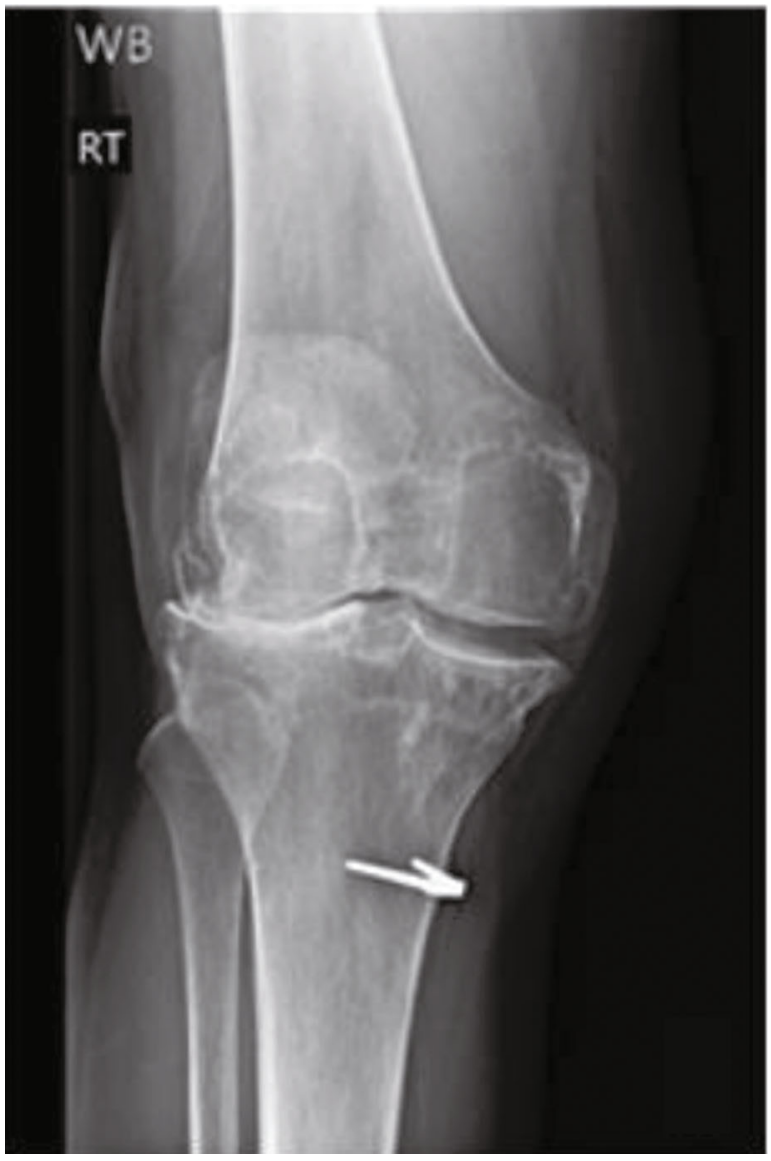

(c)

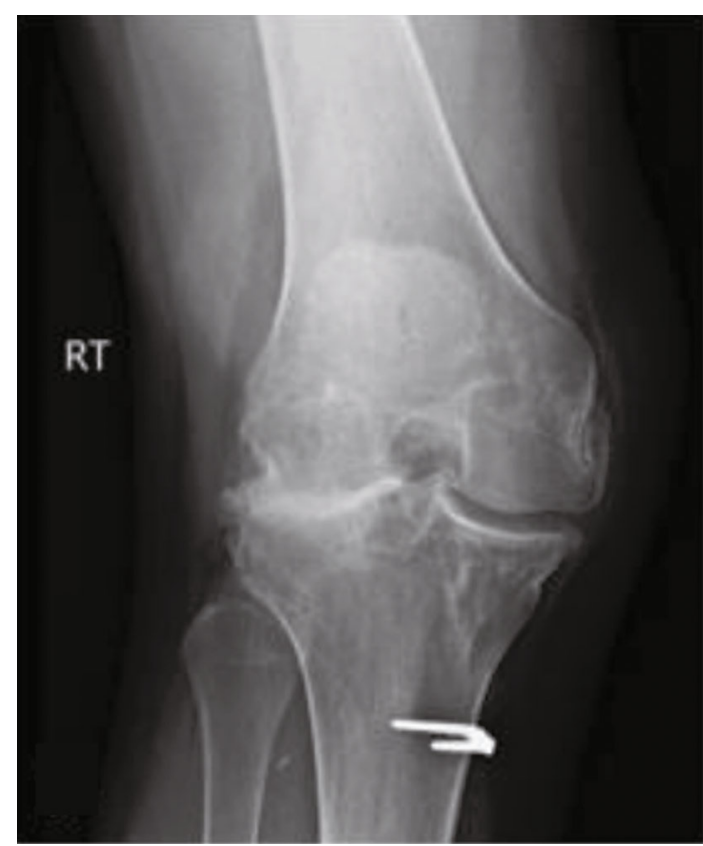

(b)

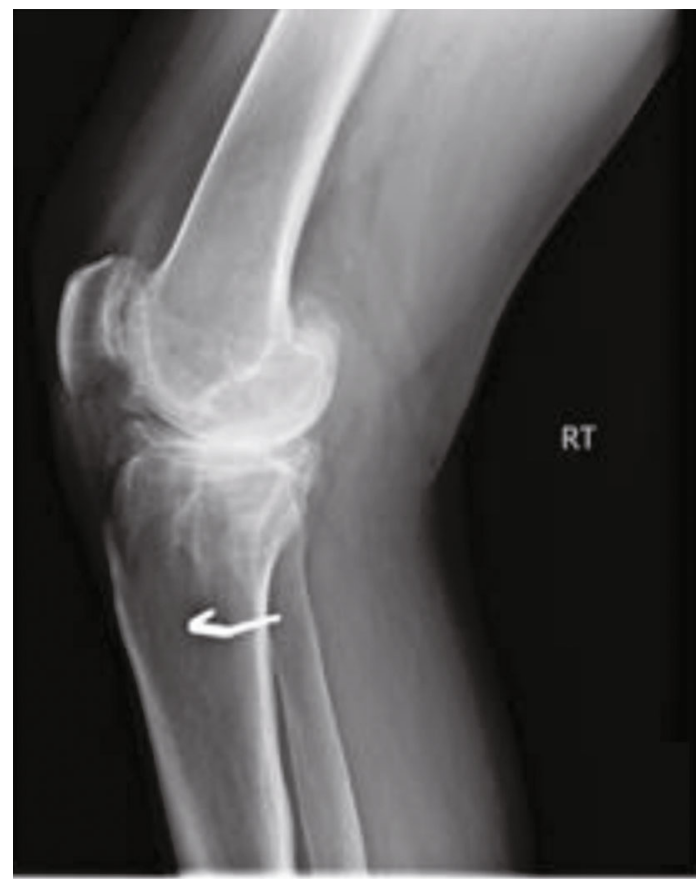

(d)

FIGURE 12: Preoperative X-ray of Case 4 showing severe osteoarthritic changes including (a) sunrise view, (b) notch view, (c) AP view, and (d) lateral view. 


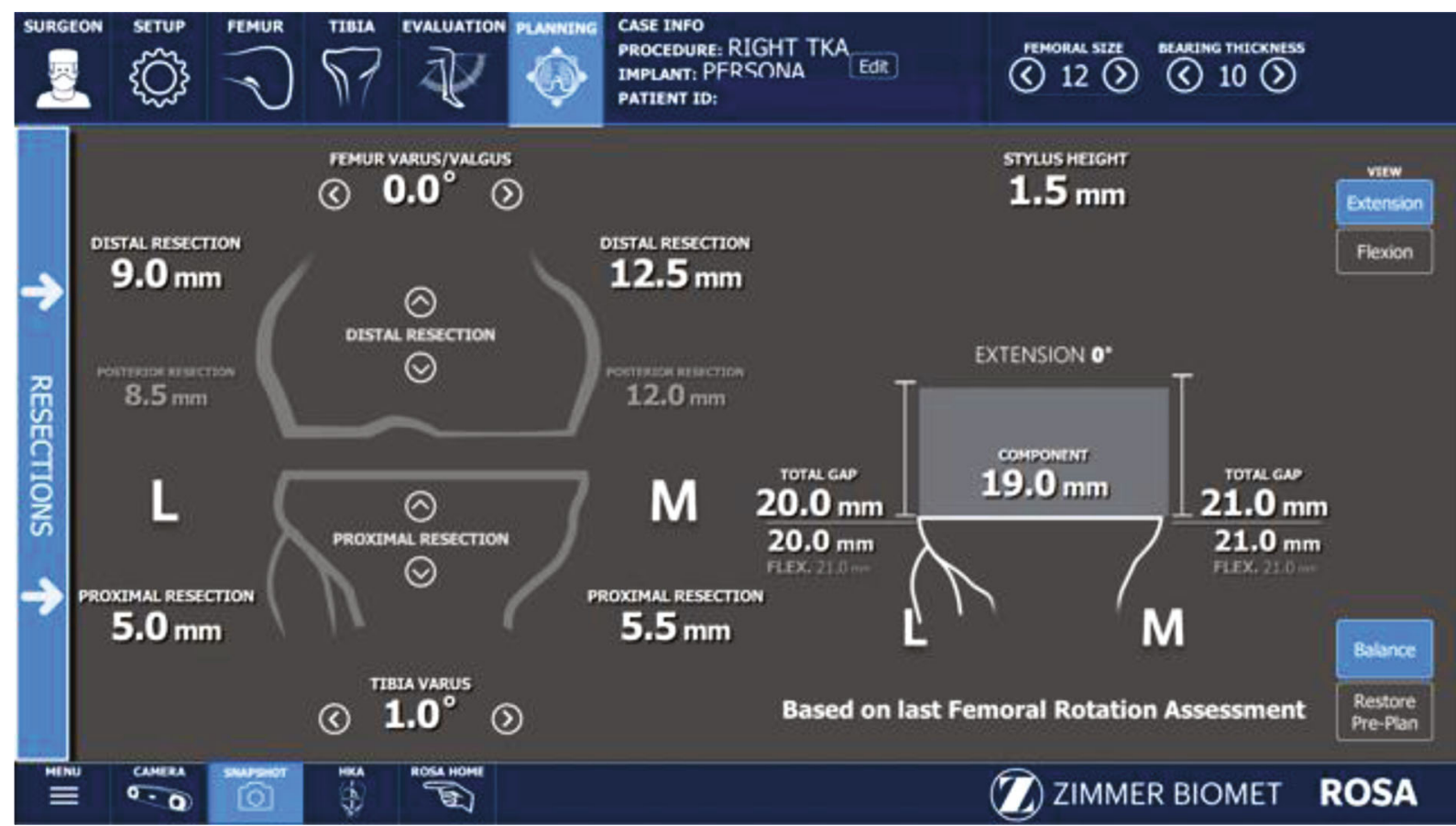

Figure 13: The generated resection plan for Case 4.

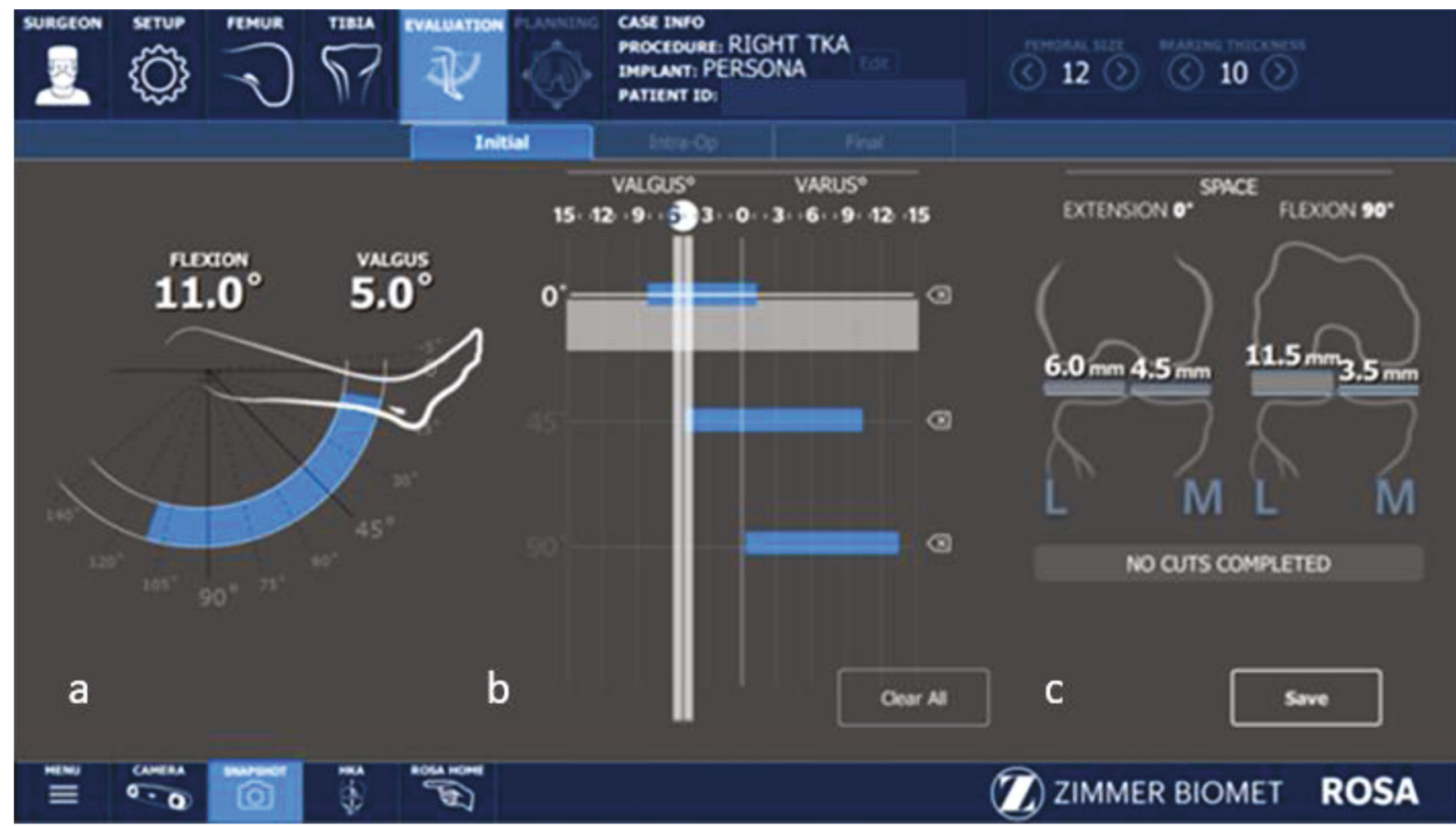

FIGURE 14: Initial evaluation of the knee for Case 4 including (a) ROM, (b) alignment, and (c) joint space evaluation.

The primary goal was to restore ligament and soft tissue balance and increase range of motion of the knee by addressing the large lateral laxity in flexion, along with balancing the knee in extension. The plan included ligament release and a bony resection as generated by the robotic application (Figure 13). To address the laxity in flexion, a larger medial distal femur resection was planned to balance the laxity and increase range of motion in flexion while decreasing the severity of the varus deformity (Figure 14). The robotic application was used to validate the cuts made in order to ensure that they were within $0.5 \mathrm{~mm}$ from the original plan (Figure 15). After the resections were validated and the TKA was complete, the application was used for a final evaluation of the knee (Figure 16). Immediately following the 


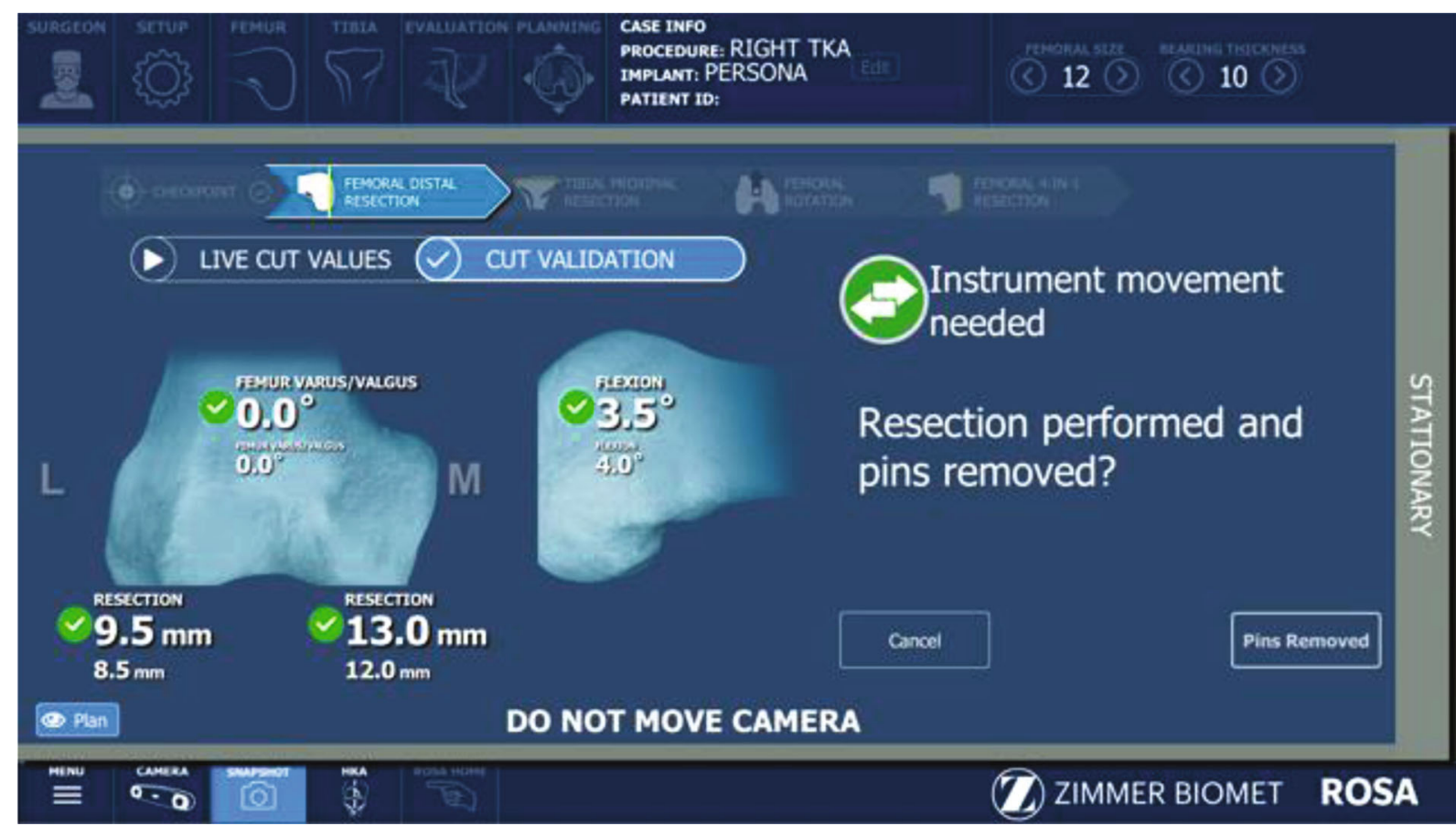

FIGURE 15: Femoral resections validated against ROSA-generated femoral resection plan.

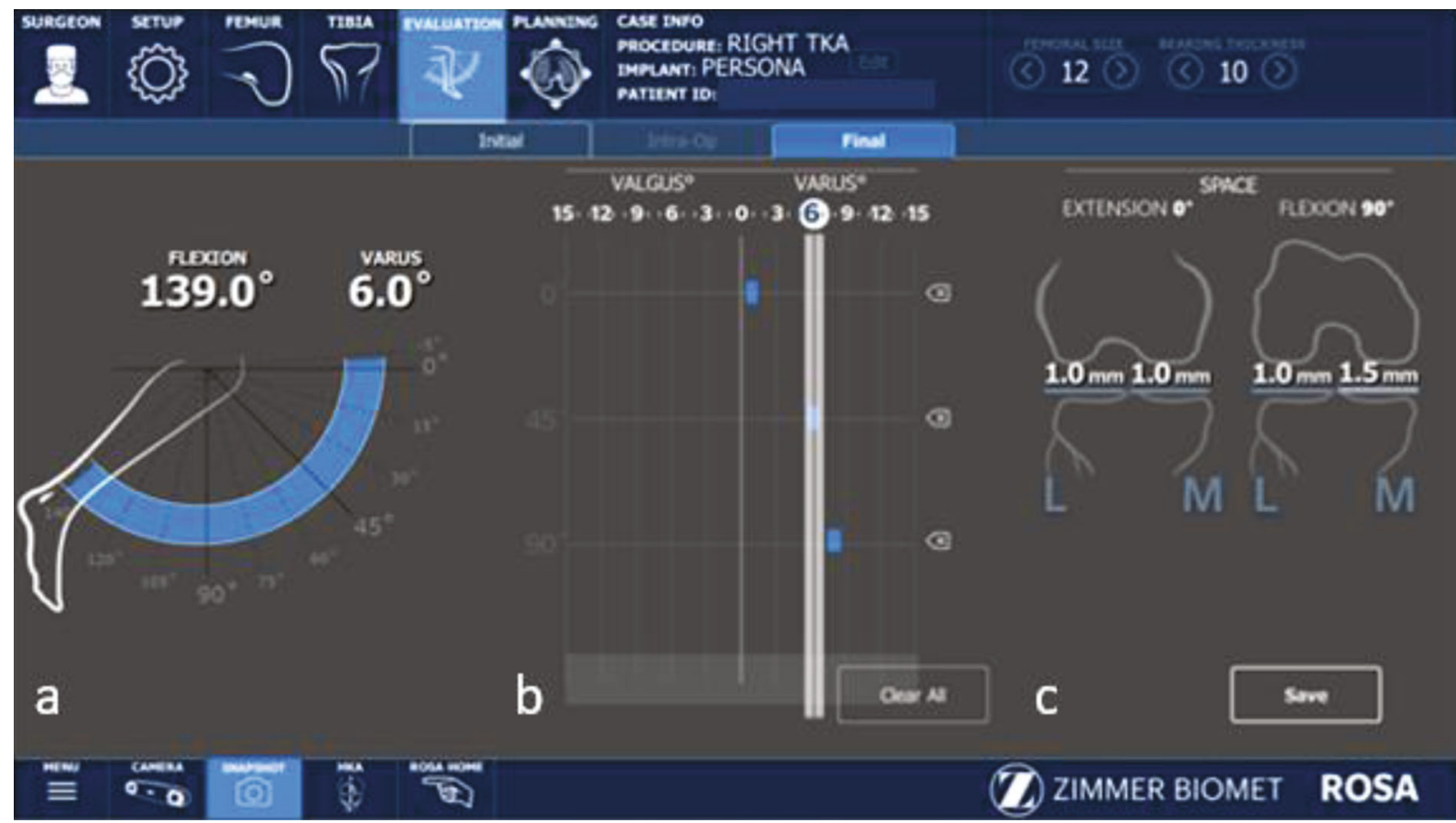

FIgURE 16: Final evaluation of the knee for Case 4 including (a) ROM, (b) alignment, and (c) joint space evaluation.

procedure, the patient was able to obtain a full arc of motion in extension and flexion (Figure 16(a)) (Table 1). Soft tissue and ligament balance were achieved by the resection plan, which reduced the medial and lateral laxity in extension and medially in flexion (Figure 16(b)). Stability was achieved by reducing the varus deformity in flexion and extension gaps to a tighter range (Figure 16(c)) (Table 2). Postoperative radiographs show an HKA of $0.9^{\circ}$ (Figure 17).
2.5. Case 5: The Complete Functionality. Case 5 highlights the complete functionality of the ROSA system. A 65-year-old female presented with severe and worsening atraumatic left knee pain and was found to have severe left knee tricompartmental osteoarthritis. Preoperative long-leg AP and lateral views of the left knee revealed Kellgren-Lawrence grade 3 changes with joint space narrowing, sclerosis, osteophyte formation, and a $16^{\circ}$ valgus alignment [16]. Additionally, a rigid 


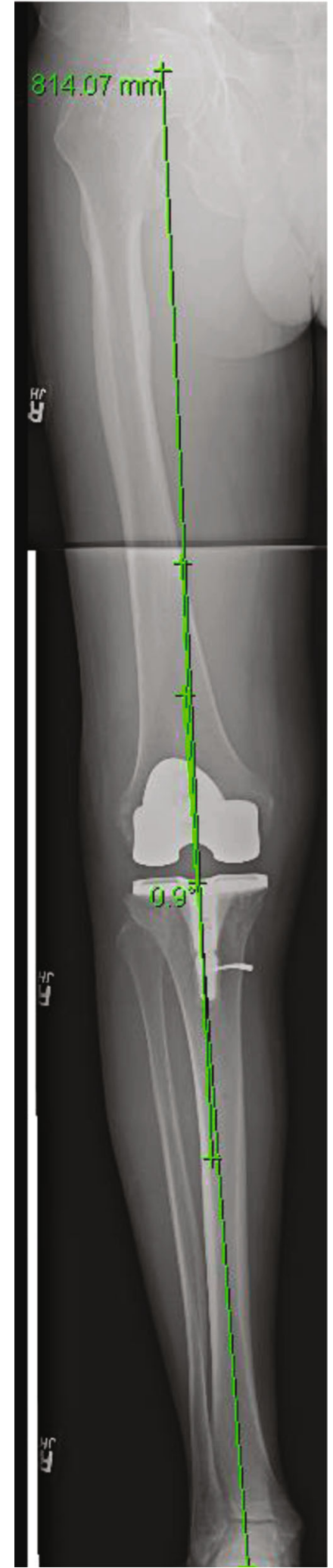

FIGURE 17: Postoperative full-leg standing radiograph of the left leg for Case 4 showing the corrected neutral mechanical alignment following TKA in 0.9 degrees if varus.

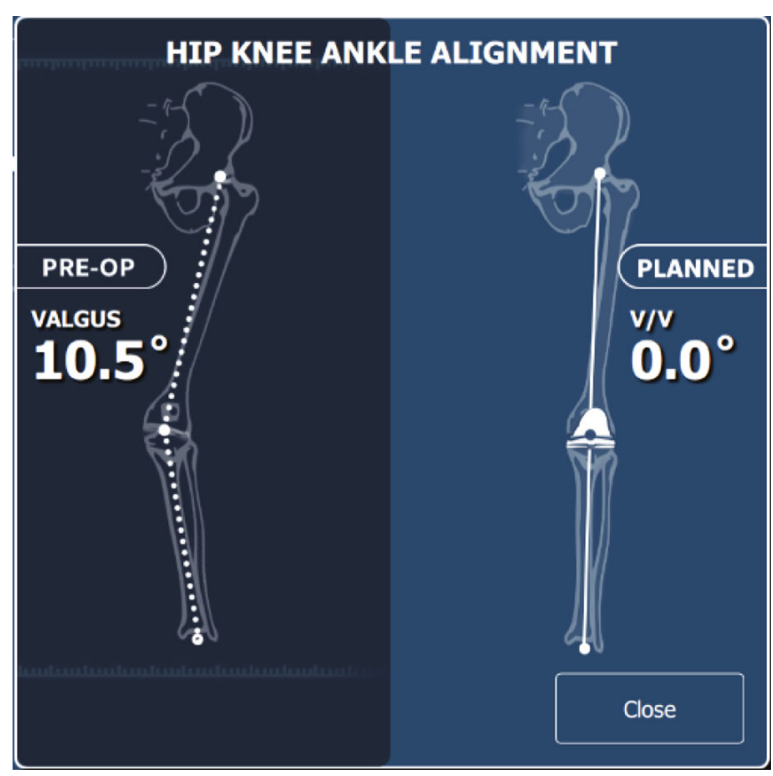

FIgURE 18: The initial evaluation (left) of 10.5 valgus in extension, and the planned HKA correction (right) for Case 5.

flexion contracture was noted on the preoperative physical exam.

Intraoperatively, the robot validated the preoperative alignment and the associated flexion contracture of the left knee. The initial preoperative alignment evaluation with ROSA showed the patient was $10.5^{\circ}$ valgus in extension (Figure 18). Additionally, a flexion contracture with a maximum extension of $23^{\circ}$ was found during the knee state evaluation (Figure 19(a)) (Table 1). The intra-op evaluation also showed that the knee was not passively correctable to a neutral alignment in extension (Figure 19(b)). However, the knee became suppler at 45 and 90 degrees, as shown by the blue shaded areas in Figure 19(b). The initial gap evaluation showed the knee was much more lax laterally in flexion than in extension (Figure 19(c)) (Table 2).

One of the major concerns of a rigid flexion contracture with associated valgus alignment is a postoperative peroneal nerve palsy. Through traditional gap balancing techniques with conventional instrumentation, additional distal femoral resections may be needed to improve extension and anticipated soft tissue releases to appropriately balance this knee replacement might be needed. While these steps allow for restoration of alignment and gap balancing, they can also increase the risk of peroneal nerve palsy and potentially create patella baja $[25,26]$.

With this novel robotic system, the ability to perform submillimeter bone resections allows the surgeon to balance the knee through boney resections versus soft tissue releases $[7,18]$. This also allows the surgeon to adjust both distal femoral and proximal tibial cuts appropriately, to help minimize the risk of patella baja, thus keeping the joint height appropriate.

The goal of this robotic assisted TKA was to correct the flexion contracture and valgus alignment, as well as to obtain the correct degree of femoral rotation, as improper femoral 


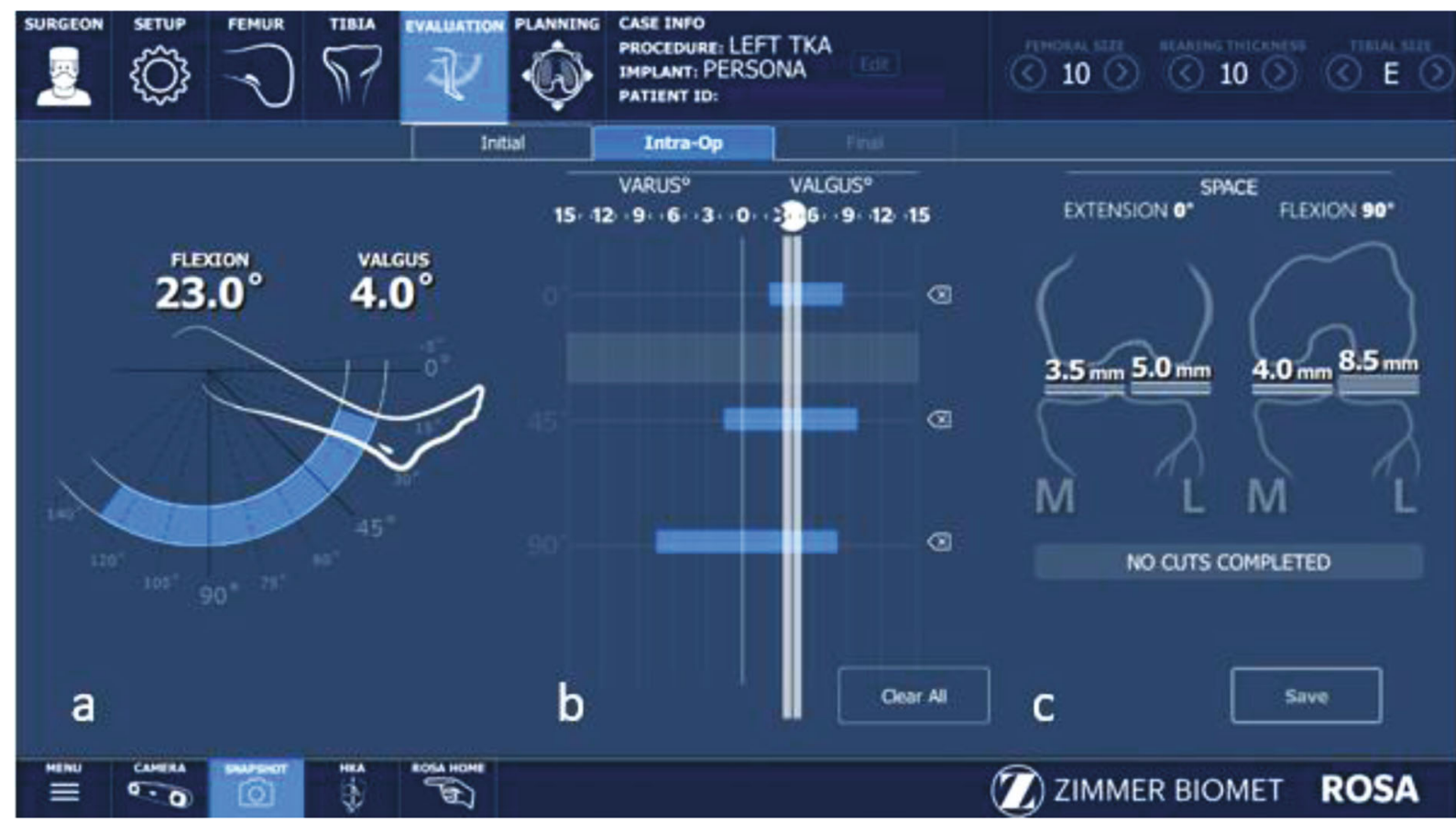

FIgURE 19: Intraoperative evaluation of Case 5 including (a) ROM, (b) alignment, and (c) joint space evaluation.

rotation can result in a trapezoidal flexion gap and poor patellar tracking. Following a gap balanced workflow, the femoral and tibial resections were appropriately performed based on preoperative soft tissue data (Figure 19).

Preoperative weight-bearing long-leg AP and lateral views (Figure 20) of the knee shows the valgus alignment measured on X-ray to be about 16 degrees. The robotic system predicted valgus alignment was 10.5 degrees (Figure 18). However, the plan was to obtain a postoperative mechanically neutral alignment (Figure 18).

With a gap balanced workflow, a successful execution of a left total knee replacement was seen (Figure 21). Range of motion was restored to 0-140 degrees (Figure 21(a)). Alignment was restored to a mechanically neutral state (Figure 21(b)), and the extension and flexion gaps were appropriately balanced (Figure 21(c)).

The coronal and sagittal balancing with ROSA was successful as indicated with postoperative extension and flexion gaps, achieving symmetry in all aspects of final knee ROM. The final gap analysis showed balanced tibiofemoral gaps both medially and laterally at 0-90 degrees of flexion and extension (Figure 21(c)) (Table 2). The comparison between the pre- and postcoronal stability assessment as shown in the blue shaded line on Figure 21(b) indicates how you can balance a knee in not only extension and flexion but also midflexion. This case was performed with a gap balance workflow utilizing ROSA, and the final knee evaluation showed appropriate correction of knee ROM, which was shown to be $0-140$ degrees within the robotic application, appropriate restoration of the femoral rotation, and successful extension and flexion gap balancing at 0,45 , and 90 degrees (Figure 21).

Postoperative X-rays show an appropriate positioned TKA (Figure 22), including appropriate restoration of the posterior condylar offset of the femur, appropriate patella tracking, and appropriate implant sizing and position. However, it is important to note that ROSA corrects the extremity alignment; as seen on the post-op short leg AP X-ray in Figure 22, the tibia appears to be in varus. However, the long-leg AP X-ray shows that the knee alignment was in fact corrected to an appropriate mechanically neutral alignment (Figure 23), showing that the ROSA plan (Figure 18) was successfully achieved clinically with postoperative X-rays.

At four months, postoperatively, the patient has reported excellent outcomes and a 95\% recovery on her VAS score. Her active knee ROM was 0-130 degrees, and she was pain free and even hiked the Grand Canyon after her 3-month post-op visit. The knee felt appropriately balanced in the clinic setting, and having the objective measures at the end of the case allowed the surgeon to have confidence that the knee was appropriately balanced. The patient also had normal postoperative neurovascular status and overall is satisfied with the result.

\section{Discussion}

Conventional TKA is one of the most successful surgeries for reducing pain and restoring function due to degenerative joint changes, yet varus-valgus errors of up to, or exceeding $4^{\circ}$, and flexion-extension errors of up to $10^{\circ}$ differences from original surgical plans have been reported [27]. This can lead to discomfort, longer or improper recovery, revision surgeries, and even implant failure [21, 22, 28-31]. Innovations in TKA are necessary to improve patient outcomes and address the proportion of patients who are dissatisfied while improving the quality and efficiency of the procedure. However, these innovations must maintain the quality of care in both routine and complex cases. As evident in the above cases, this 


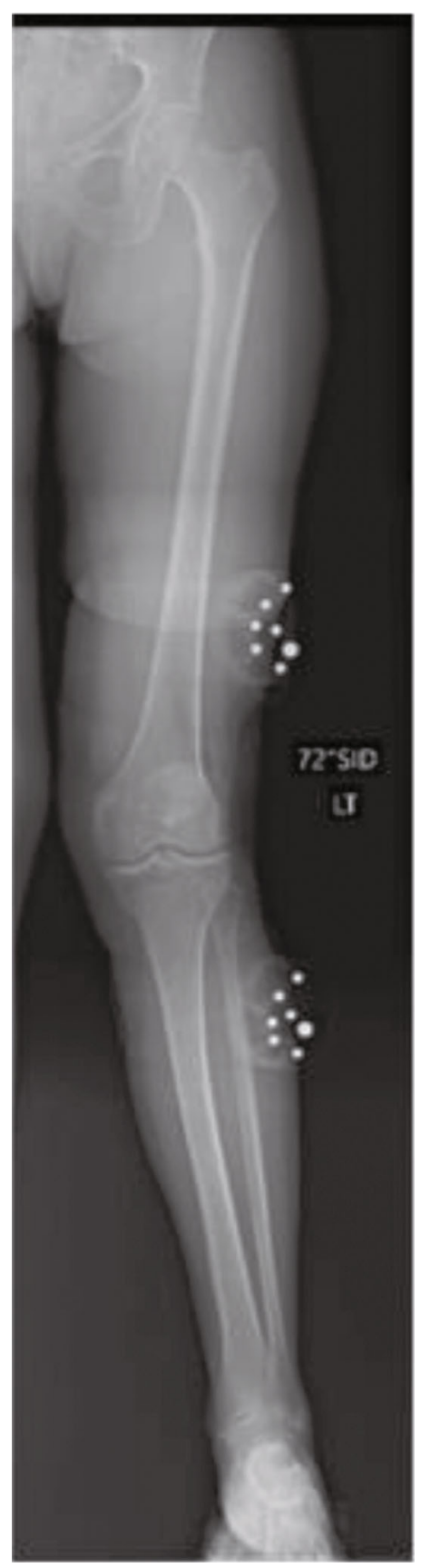

(a)

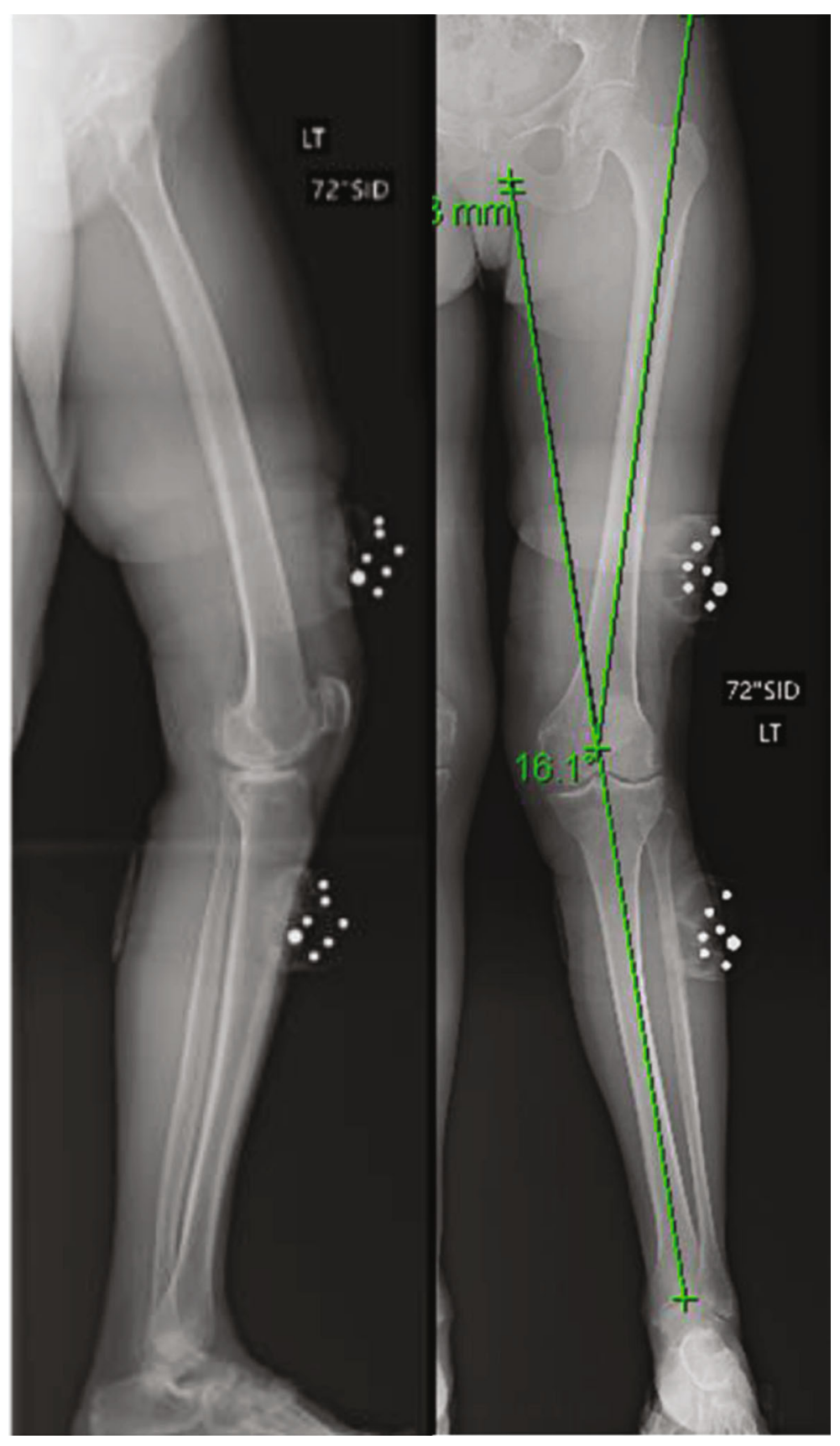

(b)

Figure 20: Preoperative long-leg (a) AP and (b) lateral X-rays showing Kellgren-Lawrence grade 3 changes and a rigid flexion contracture for Case 5. A valgus alignment in extension of 16.1 degrees, also including the markers of ROSA rigid body trackers used for preoperative 2D-3D imaging process.

novel robotic system allows the surgeon to drive every step of the case through varying degrees of deformity, turning the subjective feel of conventional knee replacement into a more objective approach in restoration of alignment, gap balancing, joint height restoration, femoral rotation, and Q-angle restoration. In order to achieve these goals, the robotic arm assists in positioning the cutting jigs in the appropriate planned cutting plane. In cadaveric studies, this robotic system has demonstrated $100 \%$ accuracy to within 3 degrees of goal for tibial and femoral coronal angles, tibial slope, and the hip-knee-ankle angle [18]. Additionally, that study demonstrated planned resections to be within $2 \mathrm{~mm}$ of goal in $>90 \%$ of the specimens reviewed. Further, compared to conventional cases in a cadaveric comparison, this system has the potential to standardize as well as to enhance the reproducibility of TKA through quantitative measures previously unavailable [7].

Similar to other robotic systems, the ability of this robotic system to provide real-time objective measurements throughout the entire TKA procedure allows the surgeon to 


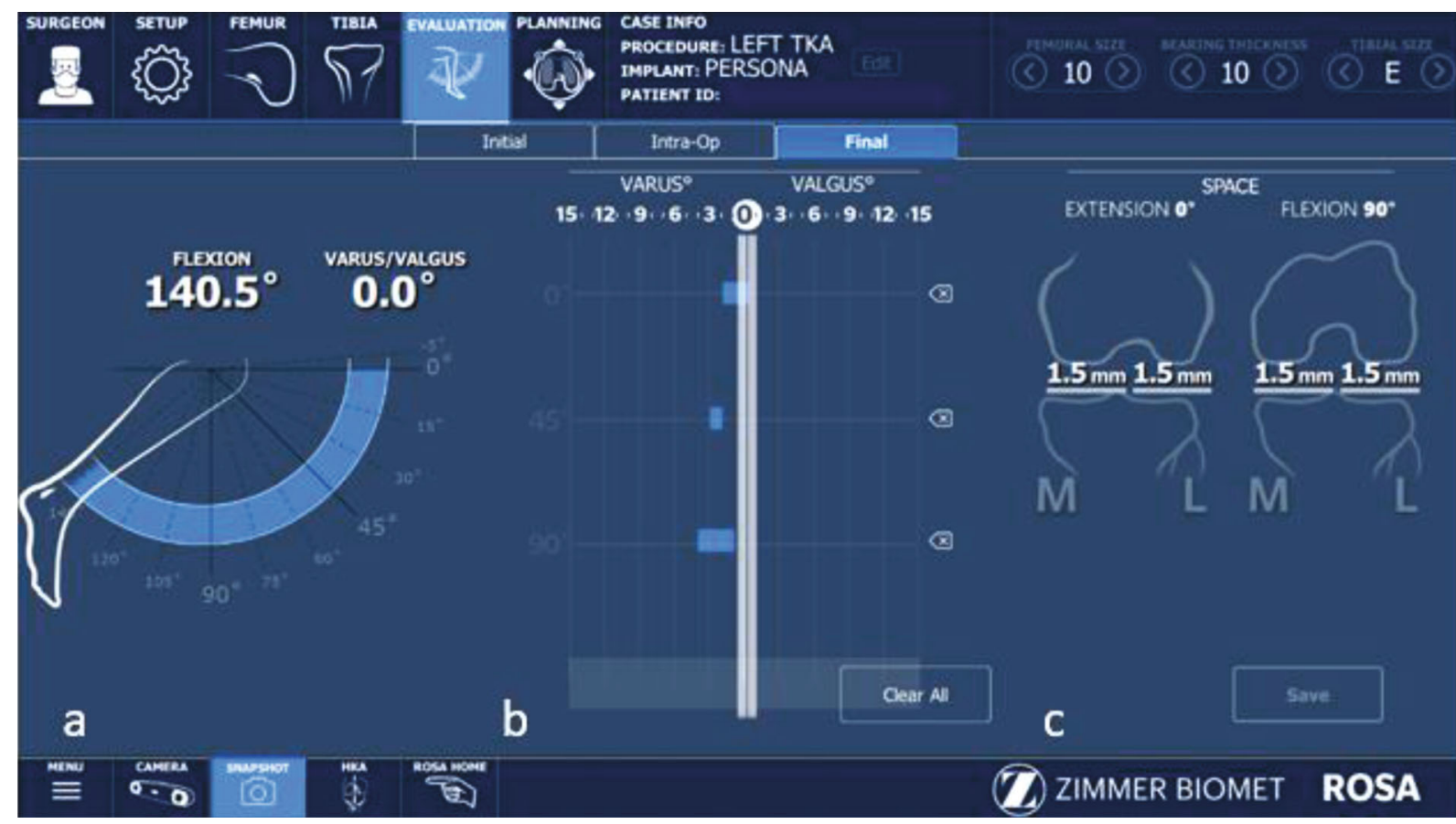

FIgURE 21: Final ROSA evaluation following TKA for Case 5 including (a) ROM, (b) alignment, and (c) joint space evaluation.

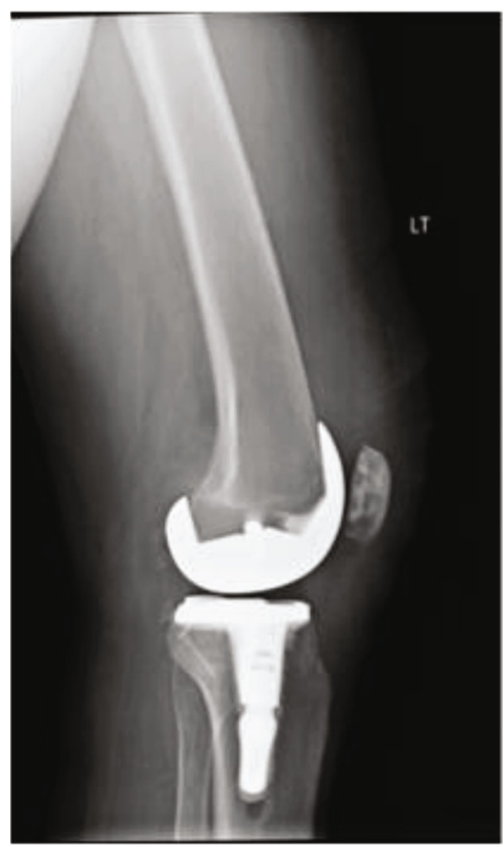

(a)
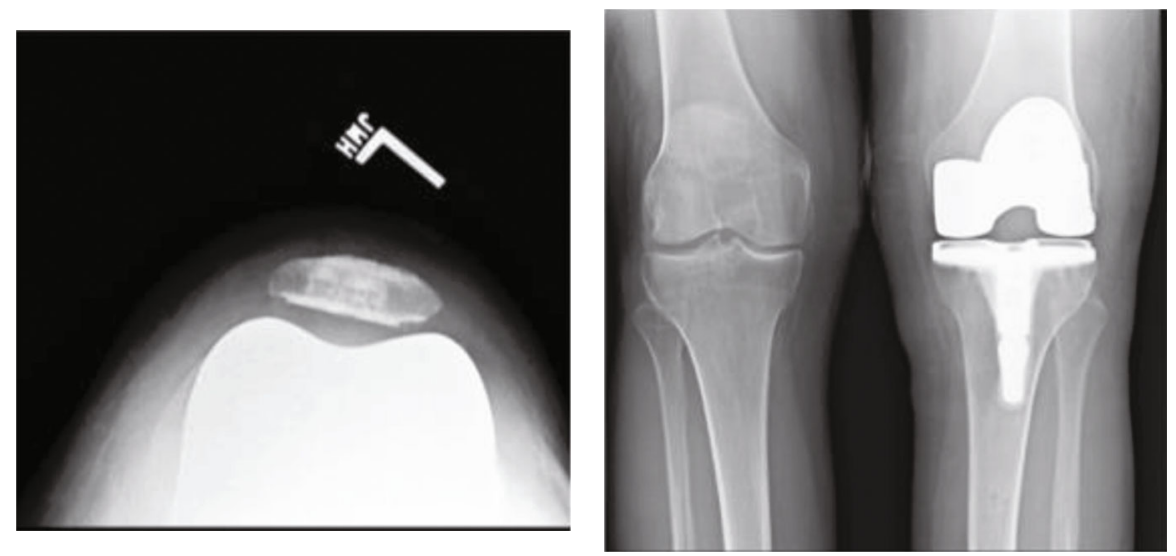

(b) (c)

Figure 22: Postoperative X-rays showing balanced gap spacing following TKA (a, c), as well as appropriate patellar tracking (b) for Case 5.

make intraoperative adjustments to the plan and provide more personalized procedures. This ensures more accurate bone resections and soft tissue balancing which are validated by the application, allowing the surgeon more confidence in adjusting the resections as needed prior to finalizing the implant. However, as with prior computer-assisted surgical (CAS) systems, robotic total knee arthroplasty may be lim- ited by the accuracy of the anatomical landmarks obtained during registration. Klasan et al. [32] have recently demonstrated some potential concerns for measurement error associated with CAS landmarks. However, robotic-assisted TKA has already been shown to be more consistent in correcting varus and valgus deformities to a range within $3^{\circ}$ of neutral mechanical alignment [33], and recent cadaveric studies 


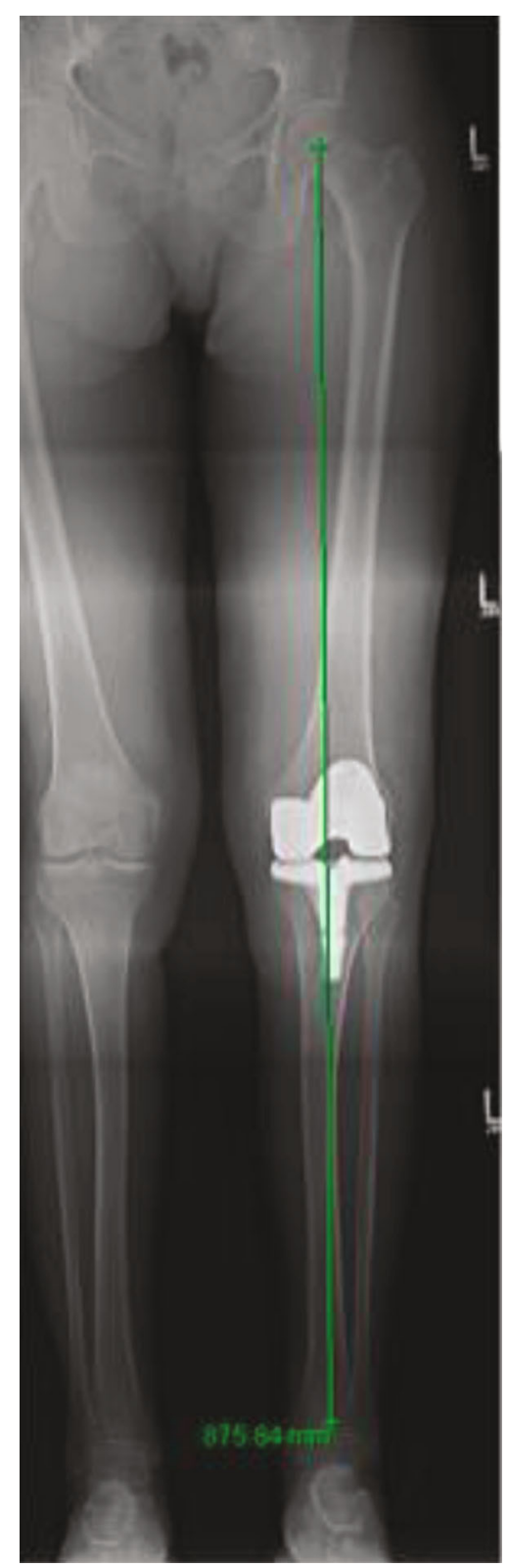

FIGURE 23: Postoperative full-leg standing radiograph of the left leg for Case 5 showing the corrected neutral mechanical alignment following TKA.

conveyed the accuracy of the ROSA total knee application at carrying out bone resection and correcting angles $[7,18]$. These studies demonstrated an average resection accuracy of $<1^{\circ}$ for all measures assessed and $<1 \mathrm{~mm}$ for resection thickness [18]. In each of the cases described, the surgeon was able to restore the knee to an appropriate functional potential compared to the initial knee evaluation, and each case highlights and addresses the functionality of this robotic system. The predicted resections were within $0.5 \mathrm{~mm}$ of actual cuts, allowing an alignment within a $3^{\circ}$ range from neutral for both extension and flexion.

This review is limited to a small case study of five patients selected for their ability to demonstrate the value of intraoperative tools available with robotic total knee arthroplasty and should be interpreted with caution as it does not provide a comprehensive review of consecutive cases. That said, the purpose of this case presentation was to demonstrate the intraoperative ability of a novel robotic system, to make patient-specific intraoperative adjustments from scientific objective data. This case series also illustrates how this system can help navigate varying degrees of deformity in total knee arthroplasty. Each case focuses on a specific aspect of TKA surgery that is important to restore at the time of surgery. Case 5 illustrates a complex primary TKA and how all goals of TKA were successfully achieved, thus providing this patient with a well-balanced, appropriately aligned, and functional TKA.

To our knowledge, this is the first supportive clinical evidence for ROSA.

\section{Summary}

These cases demonstrate the ability of the surgeon to use the objective measures provided to restore alignment, femoral rotation, gap balance, and knee ROM, through both imagebased and image-free approaches, and utilizing either a Gap Balance or Measure Resection workflow. Further research is needed to determine how and if the clinical precision of new robotic systems can be used to improve long-term outcomes, especially given the lack of literature on the system reviewed. This will require analysis of current patient outcomes and knowledge of how to prioritize different aspects of the surgery when creating patient-specific plans. Notably, there is controversy surrounding the ideal postoperative alignment, joint space, and joint obliquity. Regardless, the ability of this robotic system to provide precise intraoperative adjustments, as demonstrated by these cases, is a promising step towards more personalized and objective total knee arthroplasty.

\section{Data Availability}

The data is limited to single case reports and falls under the protected health information. For more information regarding the data availability, please reach out to the corresponding author.

\section{Conflicts of Interest}

NLC is a paid consultant for and receives institutional research support from Zimmer Biomet. ICW is a paid speaker/presenter for MicroPort Orthopedics, Pacira, and Zimmer Biomet. ICW is also a paid consultant for MicroPort Orthopedics and Zimmer Biomet.

\section{Authors' Contributions}

The authors were responsible for the final review and approval of the submitted manuscript.

\section{Acknowledgments}

This work was supported by Zimmer Biomet (Protocol \# IRU2020-21CA) and conducted with assistance by the Vail 
Summit Orthopaedic and Neurosurgery Research Foundation. The authors would like to acknowledge Mike B. Anderson, MSc, for his assistance with writing this manuscript.

\section{References}

[1] R. Gunaratne, D. N. Pratt, J. Banda, D. P. Fick, R. J. K. Khan, and B. W. Robertson, "Patient dissatisfaction following total knee arthroplasty: a systematic review of the literature," The Journal of Arthroplasty, vol. 32, no. 12, pp. 3854-3860, 2017.

[2] J. C. Chow and L. Breslauer, "The use of intraoperative sensors significantly increases the patient-reported rate of improvement in primary total knee arthroplasty," Orthopedics, vol. 40, no. 4, pp. e648-e651, 2017.

[3] L. J. Kleeblad, T. A. Borus, T. M. Coon, J. Dounchis, J. T. Nguyen, and A. D. Pearle, "Midterm survivorship and patient satisfaction of robotic-arm-assisted medial unicompartmental knee arthroplasty: a multicenter study," The Journal of Arthroplasty, vol. 33, no. 6, pp. 1719-1726, 2018.

[4] E. L. Hampp, N. Sodhi, L. Scholl et al., "Less iatrogenic softtissue damage utilizing robotic-assisted total knee arthroplasty when compared with a manual approach: a blinded assessment," Bone \& Joint Research, vol. 8, no. 10, pp. 495-501, 2019.

[5] B. Kayani, S. Konan, A. Ayuob, E. Onochie, T. Al-Jabri, and F. S. Haddad, "Robotic technology in total knee arthroplasty: a systematic review," EFORT Open Reviews, vol. 4, no. 10, pp. 611-617, 2019.

[6] L. Y. Scholl, E. L. Hampp, K. M. de Souza et al., "How does robotic-arm assisted technology influence total knee arthroplasty implant placement for surgeons in fellowship training?," The Journal of Knee Surgery, 2020.

[7] A. Seidenstein, M. Birmingham, J. Foran, and S. Ogden, "Better accuracy and reproducibility of a new robotically-assisted system for total knee arthroplasty compared to conventional instrumentation: a cadaveric study," Knee Surgery, Sports Traumatology, Arthroscopy, vol. 29, no. 3, pp. 859-866, 2021.

[8] K. Lei, L. Liu, X. Chen, Q. Feng, L. Yang, and L. Guo, "Navigation and robotics improved alignment compared with PSI and conventional instrument, while clinical outcomes were similar in TKA: a network meta-analysis," Knee Surgery, Sports Traumatology, Arthroscopy, 2021.

[9] B. Kayani, S. Konan, J. Tahmassebi, J. R. T. Pietrzak, and F. S. Haddad, "Robotic-arm assisted total knee arthroplasty is associated with improved early functional recovery and reduced time to hospital discharge compared with conventional jigbased total knee arthroplasty: a prospective cohort study," The Bone \& Joint Journal, vol. 100-B, no. 7, pp. 930-937, 2018.

[10] R. C. Marchand, N. Sodhi, H. K. Anis et al., "One-year patient outcomes for robotic-arm-assisted versus manual total knee arthroplasty," The Journal of Knee Surgery, vol. 32, no. 11, pp. 1063-1068, 2019.

[11] S. W. Jeon, K. I. Kim, and S. J. Song, "Robot-assisted total knee arthroplasty does not improve long-term clinical and radiologic outcomes," The Journal of Arthroplasty, vol. 34, no. 8, pp. 1656-1661, 2019.

[12] J. Lutzner, K. P. Gunther, and S. Kirschner, "Functional outcome after computer-assisted versus conventional total knee arthroplasty: a randomized controlled study," Knee Surgery, Sports Traumatology, Arthroscopy, vol. 18, no. 10, pp. 13391344, 2010.
[13] R. Selvanayagam, V. Kumar, R. Malhotra, D. N. Srivastava, and V. K. Digge, "A prospective randomized study comparing navigation versus conventional total knee arthroplasty," Journal of Orthopaedic Surgery, vol. 27, no. 2, article $2309499019848079,2019$.

[14] G. R. Klein, D. James, and J. H. Lonner, “Total knee arthroplasty technique: ROSA ${ }^{\circledR}$ knee," in Robotics in Knee and Hip Arthroplasty: Current Concepts, Techniques and Emerging Uses, J. H. Lonner, Ed., vol. 185, Springer International Publishing, Cham, 2019.

[15] B. Kayani and F. S. Haddad, "Robotic total knee arthroplasty: clinical outcomes and directions for future research," Bone \& Joint Research, vol. 8, no. 10, pp. 438-442, 2019.

[16] Y. Ren, S. Cao, J. Wu, X. Weng, and B. Feng, "Efficacy and reliability of active robotic-assisted total knee arthroplasty compared with conventional total knee arthroplasty: a systematic review and meta-analysis," Postgraduate Medical Journal, vol. 95, no. 1121, pp. 125-133, 2019.

[17] M. Bautista, J. Manrique, and W. J. Hozack, "Robotics in total knee arthroplasty," The Journal of Knee Surgery, vol. 32, no. 7, pp. 600-606, 2019.

[18] S. Parratte, A. J. Price, L. M. Jeys, W. F. Jackson, and H. D. Clarke, "Accuracy of a new robotically assisted technique for total knee arthroplasty: a cadaveric study," The Journal of Arthroplasty, vol. 34, no. 11, pp. 2799-2803, 2019.

[19] M. D. Kohn, A. A. Sassoon, and N. D. Fernando, "Classifications in brief: Kellgren-Lawrence classification of osteoarthritis," Clinical Orthopaedics and Related Research, vol. 474, no. 8, pp. 1886-1893, 2016.

[20] J. R. Martin, D. Levy, T. Miner, D. Conrad, J. Jennings, and D. Dennis, "Medial tibial reduction osteotomy is associated with excellent outcomes and improved coronal alignment," Reconstructive Review, vol. 7, no. 1, 2017.

[21] R. A. Berger, L. S. Crossett, J. J. Jacobs, and H. E. Rubash, "Malrotation causing patellofemoral complications after total knee arthroplasty," Clinical Orthopaedics and Related Research, vol. 356, pp. 144-153, 1998.

[22] S. J. Incavo, J. J. Wild, K. M. Coughlin, and B. D. Beynnon, "Early revision for component malrotation in total knee arthroplasty," Clinical Orthopaedics and Related Research, vol. 458, pp. 131-136, 2007.

[23] C. Y. Li, K. J. Ng Cheong Chung, O. M. E. Ali, N. D. H. Chung, and C. H. Li, "Literature review of the causes of pain following total knee replacement surgery: prosthesis, inflammation and arthrofibrosis," EFORT Open Reviews, vol. 5, no. 9, pp. 534543, 2020.

[24] D. Walmsley and D. Backstein, "Rotational alignment in total knee arthroplasty," Seminars in Arthroplasty, vol. 24, no. 3, pp. 156-159, 2013.

[25] A. Assiotis, K. To, R. Morgan-Jones, I. P. Pengas, and W. Khan, "Patellar complications following total knee arthroplasty: a review of the current literature," European Journal of Orthopaedic Surgery and Traumatology, vol. 29, no. 8, pp. 1605-1615, 2019.

[26] O. A. Nercessian, O. F. C. Ugwonali, and S. Park, "Peroneal nerve palsy after total knee arthroplasty," The Journal of Arthroplasty, vol. 20, no. 8, pp. 1068-1073, 2005.

[27] C. Plaskos, A. J. Hodgson, K. Inkpen, and R. W. McGraw, "Bone cutting errors in total knee arthroplasty," The Journal of Arthroplasty, vol. 17, no. 6, pp. 698-705, 2002.

[28] M. E. Berend, M. A. Ritter, J. B. Meding et al., "The Chetranjan Ranawat Award: Tibial component failure mechanisms in total 
knee arthroplasty," Clinical Orthopaedics \& Related Research, vol. 428, pp. 26-34, 2004.

[29] O. Furnes, B. Espehaug, S. A. Lie, S. E. Vollset, L. B. Engesaeter, and L. I. Havelin, "Early failures among 7,174 primary total knee replacements: a follow-up study from the Norwegian Arthroplasty Register 1994-2000," Acta Orthopaedica Scandinavica, vol. 73, no. 2, pp. 117-129, 2002.

[30] B. S. Lee, H. I. Cho, S. I. Bin, J. M. Kim, and B. K. Jo, "Femoral component varus malposition is associated with tibial aseptic loosening after TKA," Clinical Orthopaedics and Related Research, vol. 476, no. 2, pp. 400-407, 2018.

[31] A. J. Nedopil, S. M. Howell, and M. L. Hull, "What mechanisms are associated with tibial component failure after kinematically-aligned total knee arthroplasty?," International Orthopaedics, vol. 41, no. 8, pp. 1561-1569, 2017.

[32] A. Klasan, S. E. Putnis, S. Grasso, T. Neri, and M. R. Coolican, "Conventional instruments are more accurate for measuring the depth of the tibial cut than computer-assisted surgery in total knee arthroplasty: a prospective study," Archives of Orthopaedic and Trauma Surgery, vol. 140, no. 6, pp. 801806, 2020.

[33] J. H. Lonner and Y. A. Fillingham, "Pros and cons: a balanced view of robotics in knee arthroplasty," The Journal of Arthroplasty, vol. 33, no. 7, pp. 2007-2013, 2018. 